\title{
Altered Gene Expression in Human Brain \\ Microvascular Endothelial Cells in Response to the Infection of Influenza H1N1 Virus
}

\section{Doaa Higazy}

Cairo University

Xianwu Lin

Huazhong Agricultural University

Tanghui Xie

Huazhong Agricultural University

Ke Wang

Huazhong Agricultural University

Xiaochen Gao

Huazhong Agricultural University

Min Cui ( $\nabla$ cuimin@mail.hzau.edu.cn )

Huazhong Agricultural University

\section{Research Article}

Keywords: Blood-brain barrier, Influenza A virus, hBMECs, CNS, Neurodegenerative diseases, RNAseq

Posted Date: August 27th, 2021

DOl: https://doi.org/10.21203/rs.3.rs-850294/v1

License: (9) (i) This work is licensed under a Creative Commons Attribution 4.0 International License. Read Full License 


\section{Abstract}

Influenza viruses are not only causing respiratory illness, but also neurological manifestations were reported following acute viral infection. The Central nervous system (CNS) has a specific defence mechanism against pathogens structured by cerebral microvasculature lined with brain endothelial cells to form the blood-brain barrier (BBB). To investigate the response of human brain microvascular endothelial cells (hBMECs) to the influenza A virus, we inoculated the cells with the A/WSN/33 (H1N1) virus. We then conducted an RNAseq experiment to determine the changes in gene expression levels and the activated disease pathways following infection. The analysis revealed an effective activation of the innate immune defence by inducing the pattern recognition receptors (PRRs). Along with the production of proinflammatory cytokines, we detected an upregulation of interferons and interferon-stimulated genes, such as IFN- $\beta / \lambda$, ISG15, CXCL11, CXCL3, and IL-6, etc. Moreover, infected hBMECs exhibited a disruption in the cytoskeletal structure both on the transcriptomic and cellular levels. We also noted that pathways of neuroactive ligand-receptor interaction, neuroinflammation, and neurodegenerative diseases were noticeably induced together with a predicted activation of the neuroglia. Likewise, a number of genes linked with the mitochondrial structure and function display a significant differential expression. En masse, this data supports that hBMECs could be infected by the influenza A virus, which induces the innate and inflammatory immune response. The results suggest that the influenza virus infection could potentially induce a subsequent aggravation of neurological disorders.

\section{Introduction}

The blood-brain barrier is precisely controlling the CNS to protect neurons from the external passage of pathogens and toxins into the brain (Daneman and Prat 2015; Nassif et al. 2002). The brain microvascular endothelial cells (BMVECs) are remarkable constituents of the BBB and provide selective permeability (Rosas-Hernandez et al. 2018). Pathogens, including viruses, can damage the brain's microvascular structure and lead the BBB to lose its function and permeability, causing numerous migration of immune cells to the brain and resulting in an inflammation that could trigger several neurological brain disorders (Koyuncu, Hogue, and Enquist 2013). There is an association between peripheral and central inflammation by the passage of injury signals to the brain that initiates cytokine production or blood-borne mediators crossing to anatomically sensitive sites within the BBB (Anthony et al. 2012; Lampa et al. 2012). The respiratory system was not the only route targeted by the influenza A virus, researchers have demonstrated the virus's ability to infect the central nervous system and cause neuronal disorders (Studahl 2003; van Riel et al. 2014).

Prior investigations have implemented that influenza $A$ and $B$ viruses are linked to the incidence of mild encephalopathy with the reversible splenial lesion (MERS)(Vanderschueren et al. 2018). It was suggested that MERS is a common cause for inducing reversible lesions involved in the splenium of corpus callosum (RESLES) (Garcia-Monco et al. 2011). In one case report, MERS was a complication of influenza B primary infection for an 8-year-old girl who was not previously vaccinated (Ventresca et al. 2021). They notably observed an acute lesion in the splenium of the corpus callosum. The lesion was transient, which 
suggested that the virus's effect on the brain was reversible (Ventresca et al. 2021). Another case was reported for a 4-year-old healthy female child who suffered from influenza-associated encephalopathy (IAE); the visualized symptoms included neurological complications of temporary visual impairment and significant motor deficits (Billa et al. 2020).

However, the mechanism by which the influenza virus could induce neuroinflammation is not fully understood. Certain influenza virus strains, including A/WSN/33, were classified as neurotropic since the viral vRNA and mRNA were detected in the brain by real-time PCR following olfactory infection of mice (Aronsson et al. 2001). Interestingly, neurovirulent strains can cross to the CNS through the olfactory, vagus, trigeminal, and sympathetic nerves (Park et al. 2002). On the other hand, non-neurotropic strains such as A/PR/8/34 were suspected of inducing cognitive deterioration (Jurgens, Amancherla, and Johnson 2012). In this scenario, the activated immune response can decrease the neurotrophic (BDNF, NGF) and immunomodulatory (CD200, CXCL1) factors within the hippocampus while increasing the microglial reactivity (Jurgens, Amancherla, and Johnson 2012). Therefore, neurotropic and nonneurotropic influenza A virus strains might harm the CNS (Barbosa-Silva, Santos, and Rangel 2018). The astrocytes cells following influenza virus infection induced the flow of several proinflammatory cytokines in addition to the overexpression of genes functioning in synaptic transmission (Lin et al. 2015). IAV might aggravate multiple sclerosis coincident with CXCL5 upregulation following peripheral infection (Blackmore et al. 2017). In parallel, the increased passage of monocytes and neutrophils into the brain enhanced the transcriptomic changes of the spinal cord and cerebellum (Blackmore et al. 2017). Furthermore, the H7N7 and H3N2 are causing spine loss in the hippocampus, a slow recovery following infection, and demonstrated long-term damage to the CNS (Hosseini et al. 2018).

H5N1 causes similar pathological aspects with Parkinsonism, including loss of dopaminergic phenotype in substantia nigra pars compacta (SNpc), and alterations in number and morphology of SNpc microglia (Jang et al. 2012; Rohn and Catlin 2011). B and T cells deficient mice inoculated with H1N1 in the brainstem and hypothalamic neurons appeared to suffer from potential per se narcoleptic-like sleep disruption (Tesoriero et al. 2016). Further investigations that used chickens infected with the highly pathogenic influenza virus H7N1 suggested that the virus infects the brain endothelial cells at the early stages of $24 \mathrm{hpi}$ that subsequently disrupted the tight junctions of the BBB, and caused virus leakage into adjacent neuroparenchyma (Chaves et al. 2014). To our knowledge, this is the first research to study the hBMECs exposed to the A/WSN/1933 (H1N1) influenza virus strain. Here, we investigated that the human brain microvascular endothelial cells (hBMECs) are susceptible to influenza A virus A/WSN/33 (H1N1). The infection was accompanied by a massive alteration in gene expression associated with the production of several IFN genes and the activation of neuroinflammation signaling pathways. The neuroactive ligand-receptor interaction pathway was significantly upregulated, coinciding with the induced disruption in cell cytoskeleton and mitochondrial dysfunction on the transcriptomic level.

\section{Materials And Methods}

\section{Cells and viruses}


The human brain microvascular endothelial cells hBMECs were given generously by Dr. Xiangru Wang (Huazhong Agricultural University) and initially obtained from Prof. Kwang Sik Kim at Johns Hopkins University School of Medicine (Yang et al. 2016; Stins, Badger, and Sik Kim 2001; Stins, Gilles, and Kim 1997). The cells were cultured in a T25 flask containing Dulbecco's modified Eagle's medium (DMEM) supplemented with 10\% FBS (Gibco), 2 mM I-glutamine, 1\% MEM non-essential amino acid solution, 1 $\mathrm{mM}$ sodium pyruvate, $1 \%$ MEM amino acid solution, $1 \%$ MEM vitamin solution and $100 \mathrm{U} / \mathrm{mL}$ penicillin/streptomycin. The cells were incubated at $37^{\circ} \mathrm{C}$ under $5 \% \mathrm{CO}_{2}$ until the monolayer reach confluency. Madin-Darby Canine Kidney (MDCK) cells were used for virus titration, the cells were cultured in Dulbecco's modified Eagle's medium (DMEM; Invitrogen) supplemented with $100 \mathrm{U} / \mathrm{mL}$ penicillin/streptomycin and $10 \% \mathrm{FBS}$ (Gibco) at $37^{\circ} \mathrm{C}$ under $5 \% \mathrm{CO} 2$ incubator. A/WSN/33 (H1N1) virus strain offered by Prof. Hongbo Zhou (Huazhong Agricultural University) was expanded using 10-day-old embryonic chicken eggs, titrated, and preserved at $-80^{\circ} \mathrm{C}$.

\section{hBMECs infection with A/WSN/33 (H1N1)}

hBMECs cells were cultured in a growth medium in a 12-well plate at $37^{\circ} \mathrm{C}, 5 \% \mathrm{CO}_{2}$, and further incubated for $24 \mathrm{~h}$. We infected the cells with $7 \times 10^{6} \mathrm{PFU} / \mathrm{mL}(0.1 \mathrm{MOI})$ of A/WSN/33 (H1N1). After two hours of virus infection, the DMEM supernatant with the unbound virus was discarded, followed by three washes of PBS. Fresh DMEM with $2 \%$ FBS was added to the cells and incubated at $37^{\circ} \mathrm{C}$ under $5 \% \mathrm{CO}_{2}$. Cells were collected at different time points for RNA and protein extractions.

\section{RNA extraction and cDNA library construction}

Total RNA from the infected hBMECs with A/WSN/33 (H1N1) were collected using TRIzol (Invitrogen, NY) following the manufacturer's procedure. RNA concentration was confirmed by NanoDrop to ensure RNA quality before cDNA library construction. One $\mu \mathrm{g}$ of the total RNA was used for the BGISEQ-500 library construction, and double-stranded DNA contaminants in RNA samples were degraded by DNase I. mRNA molecules were purified from total RNA by Oligo (dT)-attached magnetic beads and fragmented into small pieces. However, N6 random primers were used for dscDNA synthesis by reverse transcription, dscDNA were subjected to end repair and, 3 ' end adenylated. Adaptors were ligated at the 3 ' end, and PCR amplification was done using specific primers. Furthermore, the PCR product was denatured into singlestranded DNA and cyclized with splint oligo and DNA ligase to process the final library. The DNB was then prepared and sequenced for SE50 (Fig. S1).

\section{RNA sequencing and annotation}

The whole sequencing process was performed by (BGI-China) following the (BGISEQ-500) platform, generating $23,761,511 \mathrm{~kb}$ of clean reads after low-quality reads removal. The data were confirmed for clean reads by FastQC for quality control, then mapped and assembled to the human reference genome GRCh38 (hg38) following the HISAT2/StringTie protocol (Kim, Langmead, and Salzberg 2015). The annotation and differentially expressed genes were obtained using the DESeq2 R package with considerable significance at a 5\% (0.05) p-adjusted value, and "apeglm" tool was used for log fold 
change shrinkage (Love 2014; Zhu, Ibrahim, and Love 2019). Differentially expressed genes were observed in a volcano plot using the R package Enhanced volcano (Blighe K 2020). Gene ontology GO, KEGG, and GSEA resulted from the $\mathrm{R}$ package clusterProfiler and the $\mathrm{R}$ package DOSE (Yu et al. 2012; $\mathrm{Yu}$ et al. 2015). The figures were visualized using the $R$ package enrichplot and ggplot2 (Yu 2019; Wickham 2009). The differentially expressed genes were also uploaded to the database of InnateDB to enrich the innate immune-related functions (Breuer et al. 2013). The canonical pathway, upstream regulators, and network analysis were generated through IPA (Ingenuity Pathway Analysis, QIAGEN Inc.).

\section{Western blotting}

After 12 hours of infection, hBMECs cells were washed twice with ice-cold phosphate-buffered saline (PBS) and collected using radioimmunoprecipitation assay (RIPA) containing protease inhibitor cocktail (Roche) and phosphatase inhibitor cocktail (Roche). The cells were homogenized using a sonicator machine (Qsonica LCC, USA), followed by centrifugation at $10,000 \mathrm{~g}$ for $10 \mathrm{~min}$ at $4^{\circ} \mathrm{C}$. The cell debris was discarded, and the protein concentration was measured with a BCA protein assay kit (Beyotime, China). Moreover, the protein was electrophoretically separated on a $10 \%$ sodium dodecyl sulfatepolyacrylamide gel electrophoresis (SDS-PAGE). We then transferred the proteins to polyvinylidene difluoride (PVDF) membranes $0.22 \mu \mathrm{m}$ (Bio-Rad, CA). The membrane was soaked in $5 \%$ non-fatcontaining milk in Tris-buffered saline with $0.1 \%$ Tween 20 and blocked for two hours at room temperature. It was then incubated overnight with the primary polyclonal anti-rabbit NP protein (GeneTex Inc., CA, USA), and rabbit anti- $\beta$-actin primary antibodies (Proteintech, China) at $4^{\circ} \mathrm{C}$ (as a loading control). After washing with TBST, the membranes were incubated with a goat anti-rabbit secondary antibody for one $h$ at room temperature. Finally, all the signals were visualized using Chemiluminescent chromogenic substrate ECL.

\section{Real-Time Quantitative RT-PCR (qRT-PCR)}

Total RNA of hBMECs was isolated using TRIzol (Invitrogen, Grand Island, NY, USA), the RNA was reverse-transcribed into cDNA by 5X All-In-One RT MasterMix (abm, Canada). The qPCR was performed using RealUniversal Color PreMix SYBR Green (Tiangen, China) in ABI ViiA7 PCR system (Applied Biosystems, Foster City, CA, USA). qPCR was performed to evaluate the transcriptional levels of host response genes based on RNAseq data analysis. Expression was normalized to the $\beta$-actin reference gene levels, while relative expression was calculated using the comparative method of $2-\Delta \Delta \mathrm{Ct}$. The virus replication curve was quantified and normalized by using virus copy numbers (Rüdiger et al. 2019; Frensing et al. 2016). Changes in gene expression were examined by t-test, and $p<0.05$ was considered significant. All primers manipulated in this study are listed in (Table S1).

\section{Immunofluorescence and cell morphology}

The cell morphology was observed during different time points after infection using phase contrast (Ph) Microscopy by Nikon inverted microscope Ti-U (ECLIPSE, Japan). hBMECs cells were seeded in a density of $1.5 \times 10^{4}$ per well on chamber slides and deposited at the bottom of a 24-well plate. The cells were 
inoculated with $\mathrm{A} / \mathrm{WSN} / 33(\mathrm{H} 1 \mathrm{~N} 1)$ at $0.1 \mathrm{MOI}$ and incubated at $37^{\circ} \mathrm{C}$ at $5 \% \mathrm{CO}_{2}$ for $12 \mathrm{~h}$. The slides were rinsed three times with $1 \times$ PBS and further fixed with $4 \%$ paraformaldehyde (PFA) for 10 minutes at $37^{\circ} \mathrm{C}$. After washing with PBS, the cells were permeabilized in $0.1 \%$ Triton X-100 in $1 \times$ PBS at room temperature for 10 minutes, then washed again with PBS. Permeabilized cells were then blocked with $2 \%$ BSA in PBS for 1 hour at room temperature and washed. For immunofluorescence, the cells were incubated with the primary anti-nucleoprotein NP (1:500; rabbit polyclonal, GeneTex Inc., CA, USA) diluted in 0.1\% BSA and incubated for three hours at room temperature. The secondary antibody used was Alexa flour 647 Goat Anto-Rabbit IgG (1:500; Thermofisher). After washing, the cells were stained for F-actin with fluorescent FITC-conjugated Phalloidin for 2 hours at $37^{\circ} \mathrm{C}$ and with Hoechst for 5 minutes. The slides were observed under a laser confocal microscope (Leica, Germany).

\section{Statistical analysis}

The experiments were performed in triplicate and repeated three times with similar results. The values were shown as the mean \pm standard error of the mean (SEM), and the statistical significance of the differences between groups was determined by Tukey's post hoc tests and student's t-test a $p<0.05$. The statistical analysis of differential gene expression profiles was done using $\mathrm{R}$ software (version 3.6.3). The HISAT2 alignment tool was used for mapping RNA sequencing reads to the reference genome. StringTie assembler tool of RNAseq alignments was used, which uses a novel network flow algorithm as well as an optional de novo step of assembly. The StringTie's output was processed by the DESeq2 R package to obtain the differentially expressed genes. Genes were listed when considering acceptance of significance at less than 0.05 of adjusted $p$-value and a $\log _{2}$ fold change (Log2FC) of \pm 1 . The volcano plot was observed using the enhanced volcano plot R package with a cutoff for Log2FC at \pm 2 and 0.05 of an adjusted $p$-value. The $\mathrm{R}$ package ClusterProfiler was used with its two methods, gene set enrichment analysis (GSEA) and overrepresentation analysis for the hypergeometric test. The enrichment analysis was applied to the differentially expressed genes obtained from the DESeq2 analysis to determine the genes' functional distribution to GO terms and KEGG pathways using a cutoff for the $p$-adjusted value at

0.05. The figures were visualized with two R packages enrichplot and ggplot2. The canonical pathway, upstream regulators, and network analysis were generated through IPA (QIAGEN Inc.). IPA conducts core analysis using two statistical outputs. First, $p$-value derived from a Right-Tailed Fisher's Exact test to reflect that the estimated association or overlapping between a set of significant molecules and a pathway or function that might be a result of random chance (The smaller the $p$-value, the likely the random association exists), which finally does not consider the directional effect. The second output is the z-score, or standard score, which is the number of standard deviations a given data point lies above or below the mean (Kramer, Green, and Pollard Jr 2014). A positive z-score indicates that the raw score is higher than the mean average (increases); a negative $z$-score reveals the raw score is below the mean average (decreases). Graphs are plotted and analyzed by GraphPad Prism software version 8 (GraphPad, La Jolla, CA, USA). All figures were conducted in Adobe Illustrator (Adobe, Mountain View, CA, USA).

\section{Results}




\section{Influenza A Virus Invades hBMECs}

To assess the infection of A/WSN/33 in hBMECs, we inoculated the cultured cells with influenza A virus $\mathrm{A} / \mathrm{WSN} / 33$ strain at $\mathrm{MOI} 0.1$. The cells were monitored with phase contrast $(\mathrm{Ph})$ microscopy for morphological changes. The cytopathic effect became dramatically obvious during $24 \mathrm{hpi}$ and $48 \mathrm{hpi}$ (Fig. 1a). The influenza A virus nucleoprotein (NP) gene expression was detected over different time points of infection by RT-qPCR that significantly overexpressed at $6 \mathrm{hpi}$ and continued to increase till 48 hpi (Fig. 1b). We collected the supernatant, and the virus titer was around $10^{2} \sim 10^{3}$ (data not shown). Simultaneously, the translated nucleoprotein was recognized by western blot shortly at $6 \mathrm{hpi}$ and showed high protein intensities at $24 \mathrm{hpi}$, and $48 \mathrm{hpi}$ (Fig. 1c).

The Influenza virus can shuttle between the nucleus and cytoplasm. Hence, the pivotal role of the influenza virus NP is to encapsidate the virus genome for transcription, replication, and packaging, in addition to its ability to interact with cellular polypeptides, including actin (Portela and Digard 2002; Terrier et al. 2016). The hBMECs were seeded in a 24-well plate and infected with A/WSN/33 at $0.1 \mathrm{MOI}$. After $12 \mathrm{~h}$, the cells were fixed, permeabilized, and blocked. The cells were stained for the anti-NP (red), Factin (green), and nucleic acids (blue), and further visualized by laser confocal microscopy (Fig. 2). The results indicated that $\mathrm{H} 1 \mathrm{~N} 1$ could hijack into hBMECs and find its way to the cell nucleus, while the intact cells show no signal to the virus NP. Briefly, the virus NP appeared to accumulate in the cell nucleus and to slightly colocalize with actin filaments. The filamentous actin cytoskeleton showed a re-organization and disruption within infected cells. It appears to be more thickened and displays an irregular doughnut shape compared to the intact cells. We also noticed a granule-shaped virus NP in the cytoplasm and near the edges of the plasma membrane. These data demonstrate that hBMECs are highly susceptible to influenza A virus invasion.

\section{Altered Gene Expression in Infected hBMECs}

We performed a total RNA extraction at $12 \mathrm{~h}$ following the incubation with A/WSN/33 (H1N1). The cDNA library was constructed then sequenced by the BGISEQ-500 platform. The data were mapped, assembled, and annotated by HISAT2/StringTie and DESeq2, respectively. Outwardly, the differentially expressed genes (DEGs) proclaim a significant alteration for the mRNA of the infected hBMECs compared to the non-infected cells. The analysis is listing a total of 5,500 differentially expressed genes, among which 3,712 were upregulated, and 1,788 were down-regulated ( $|\log 2 \mathrm{FC}| \geq 1 ;$ padj $<0.05)$, the DEGs of infected hBMECs are available and listed in Supplementary file 2. The volcano plot (Fig. 3a) displays the genes of infected hBMECs at $12 \mathrm{~h}$ post-infection with ( $|\log 2 \mathrm{FC}| \geq 2 ;$ padj< 0.05 ). In comparison, the heatmap (Fig. 3b) points out the top 20 significant genes expressed in infected hBMECs compared to intact cells. Interestingly, genes of antiviral activity were induced in parallel to the interferon stimulating genes (ISGs), including interferon-induced proteins with tetratricopeptide repeats (IFITs) and interferoninduced protein such as IFI44 that were all significantly upregulated following H1N1 infection. Several regulatory genes such as DHX58 (the Probable ATP-dependent RNA helicase), ISG15 (Interferonstimulated gene 15), IRF7 (Interferon regulatory factor 7), IL10 (Interleukin 10), and TRIM15 (Tripartite 
Motif Containing 15) were associated with type I interferon production also increased dramatically. On the other hand, type III interferon gene IFN- $\lambda 1$, IFN- $\lambda 2$, and IFN- $\lambda 3$ were highly upregulated in gene expression than type I interferon IFN- $\alpha$ and IFN- $\beta$, along with the over-expression of other cytokines, including CXCL2, CXCL3, CXCL8, CXCL10, CXCL11, and CXCL16. The results indicate a significant alteration in the mRNA level for hBMECs infected with WSN.

\section{Enrichment Analysis Predicts Enhanced Disruption of the Cells' Cytoskeletal Structure}

The R package clusterProfiler identified the GO and KEGG enriched terms based on gene set enrichment analysis, while the network analysis and canonical pathways were generated by IPA. The GO-based GSEA implied 1,691 significant GO terms (padj $\leq 0.05$ ), among which 223 terms belong to cellular components, 1,226 to biological processes, and 242 to molecular functions. Whereas the KEGG-based GSEA significantly enriched 57 pathways (padj $\leq 0.05$ ). Enriched GO and KEGG terms are listed in the supplementary data (see supplementary files 3,4 ). The top 10 activated and suppressed GO terms are visualized in a dot-plot (Fig. 4) with dot size reflecting each term's gene counts. Within the top 10 activated GO terms, the majority of enriched genes belong to the 'plasma membrane' (G0:0005886), 'extracellular region' (G0:0044421), and 'immune system process' (G0:0003008). Whereas, among the most significant suppressed GO terms were 'mRNA modification' (G0:0016556) and 'establishment of mitotic spindle orientation' (GO:0040001). Interestingly, the GSEA based GO analysis revealed a negative running enrichment NES=-2.3 score of 52 genes participating in the 'Regulation of microtubule cytoskeleton organization' (GO:0070507) (Fig. 5a, b), and a positive running enrichment score NES=1.31 of 185 genes activating the 'actin cytoskeleton organization' (G0:0030036) (Fig. 5c, d). The downexpressed genes include GNAI1 (G Protein Subunit Alpha I1), PSRC1 (Proline And Serine Rich Coiled-Coil 1), CEP131 (Centrosomal Protein 131), RASSF7 (Ras Association Domain Family Member 7), and CCNF ( Cyclin F ) (Fig. 5b). However, ACTC1 (Actin Alpha Cardiac Muscle 1), MYOC (Myocilin), BST2 (Bone Marrow Stromal Cell Antigen 2), TACSTD2 (Tumor-Associated Calcium Signal Transducer 2), and CSF1R (Colony Stimulating Factor 1 Receptor) were among the top overexpressed genes (Fig. 5d). Likewise, the RhoGDI signaling pathway (Fig. S2) showed a significant downregulation (Inhibition) in hBMECs infected with WSN (z-score=-3.2), which is known for its critical role in the organization of actin cytoskeleton. The pathway displays an activated level of the F-actin. Simultaneously, the ERM and Rho families known to function as cytoskeletal linkers and key regulators to the actin cytoskeleton were proposed to downregulate. Consistent with our previous result (Fig. 2), we observed that during WSN infection, a considerable number of genes that control the cytoskeleton and cell mobility are dysregulated in hBMECs.

\section{The Innate Immune and Inflammatory Response of hBMECs to Influenza A virus}

For more details, the DEGs list was uploaded to InnateDB to obtain an over-representation analysis (ORA) for innate immune-related GO. The highest significant $6 \mathrm{GO}$ terms of cellular components, biological processes, and molecular functions were observed in a pie chart according to their corresponding gene counts (Fig. 6). There is a high ratio of gene changes were found to participate in the 
ribosome and cytosolic ribosomal subunits of cellular components. Accordingly, the enrichment map generates a cluster of enriched biological processes with similar genes overlapped, yielding a dense interaction network between a set of $\mathrm{GO}$ terms. The network links multiple $\mathrm{GO}$ terms, including the plasma membrane, immune effector process and centered with the immune system process (Fig. S3a). Consequently, the findings of the functional enrichment analysis of (Fig. 4) and (Supplementary Fig. 3a) in addition to the ridge-plot of (Fig. S3b) illustrate that the immune and inflammatory responses are highly integrated into the biological changes associated with hBMECs infection with the WSN influenza virus at $12 \mathrm{hpi}$.

On the other hand, the GSEA based KEGG depicts the top significant 20 pathways, among which ten are activated, while the others are suppressed (Fig. 7). Particularly, a high number of the upregulated DEGs were predominantly enriched in the 'cytokine-cytokine receptor interaction' pathway (NES $=1.89$, padj=0.0001), (Fig. 8a, b) and 'neuroactive ligand-receptor interaction' pathway (NES=1.7, padj=0.0003), (Fig. 8c, d). The ridge-plot is visualizing the expression distributions of core enriched genes for the enriched categories of GSEA, interpreting the pathways responding to the viral infection. Such as the 'viral protein interaction with cytokine and cytokine receptor', 'JAK-STAT signaling', 'RIG-I-like receptor signaling', and 'NOD-like receptor signaling' pathways that were all activated, while 'DNA replication' and 'RNA degradation' were down-regulated (Fig. S4a). Likewise, the upset plot visualizes the genes overlapping in different gene sets by plotting the fold change distributions with various categories (Fig. S4b).

Viruses activate the innate immune system through the PRRs, including the Toll-like receptors, RIG-I-like, NOD-like receptors, and C-type lectin receptors (Amarante-Mendes et al. 2018). They also initiate the antiviral immune response by inducing the transcription of interferon and inflammatory molecules. IPA canonical pathways indicate the activation of PRRs, interferon signaling, TREM1 signaling, and neuroinflammation signaling pathway (Fig. S5a). The interferon signaling pathway (Fig. 9a, z-score=4.2) indicates the induction and overexpression among its downstream cascade, including ISGs, IFITs, and IFIs. We performed qPCR of the type III IFN- $\lambda 2 / 3$ (Fig. 9b) at different infection time points to further support our results. The transcriptional activity of the mRNA significantly increased during the 6 to $12 \mathrm{hpi}$ and dramatically decreased at 24 hpi. However, type I IFNs can actively induce ISG15 up-regulation (Fig. $9 \mathrm{c})$. IFN- $\beta$ mRNA was significantly increasing from 12 hpi to reach its peak, recording a 500 -fold change compared to the control hBMECs at $48 \mathrm{hpi}$ (Fig. 9d).

Moreover, IPA upstream regulator analysis was conducted to identify the genes that might work as regulators for the underlined DEGs in our dataset and predict whether they are activated or inhibited. IFN$\beta$ has been significantly upregulated in our dataset, and it also functions as a positive upstream regulator within IPA analysis. The regulatory network of IFN- $\beta$ (Fig. S5b, z-score=5.6) predicts to have a downstream effect on activating the proinflammatory cytokines, including TNF, IL-6, and IL-1 $\beta$ and several types of ISGs while underlying the inhibition of TGFBR1 and CLK2. Similarly, INFL1 (Fig. S6, zscore=5) appears to act as a significant upstream regulator to several cytokine genes. The mechanistic network (Fig. 9e) of IFN- $\beta$ shows a plausible set of connected upstream regulators contributing to gene 
expression changes observed in our dataset. It predicts the activation of cell surface receptor IFNAR that has further activated STAT1, STAT2, and STAT3 transcription factors. While the regulator effects demonstrate the methodology by which the activated upstream regulator IFN- $\beta$ and its downstream effects might cause a potential inhibition to the 'replication of viral replicon' (Fig. S7). Briefly, the WSN virus infection strongly induces the innate and inflammatory immune response of hBMECs.

\section{Alteration in the Mitochondrial Molecular Function}

We are now curious if the hBMECs mitochondrial system could be affected by WSN. Several genes associated with mitochondrial functions have developed significant expression changes in the transcriptomic analysis, some of which have been confirmed by qPCR (Fig. 10a).

On the other hand, the NDUFS2 (NADH: Ubiquinone Oxidoreductase Core Subunit S2), when mutated, causes dysfunction in the mitochondrial complex I, while the UQCRFS1 (Ubiquinol-Cytochrome $\mathrm{C}$ Reductase, Rieske Iron-Sulfur Polypeptide 1) is inherently functioning in the mitochondrial complex III. The SDHA (Succinate Dehydrogenase Complex Flavoprotein Subunit A) gene encodes a major catalytic subunit of succinate-ubiquinone oxidoreductase. The three genes mentioned above are considered to be mandatory in the respiratory chain. And exhibiting a dramatic decrease in their expression might exacerbate the incidence of respiratory chain dysfunction, which is common with neurodegenerative diseases such as Alzheimer's and Parkinson's. Additionally, Caspase14 (CASP14) induced following an apoptotic stimulus, along with CASP10, CASP6, and CASP8 of DEGs, were all deregulated and implicated to principally participate in the programmed cell death. Withal, the running enrichment of the 'mitochondrial protein complex' (GO:0098798) and 'mitochondrial gene expression' (G0:0140053) are both negatively scored (NES = -2.4, padj=0.02), (NES=-4.2, padj=0.009) (Fig. 10b,c and Fig. 10d, e). The data above and the GSEA indicate that mitochondria could have played a role in hBMECs disruption during influenza A virus infection.

\section{Neurodegeneration Induced Pathways in WSN Infected hBMECs}

The above results showed an inflammatory response triggered by virus infection to hBMECs, mainly by activating PRRs interaction, interferon signaling, and neuroinflammation signaling pathways followed by the production of several interferon stimulating genes (Fig. S5a). The IPA regulatory effect suggests that the inflammatory induced $\mathrm{F} 2$ gene might be involved in neuroglia activation, mainly through mediating its targets, including FN1, IL1B, CXCL10, TNF, NOS3, SERPINE1, IL6, MIF, ADIPOQ, CXCL8, and CX3CL1 (Fig. 11a). Since neuroinflammation is highly associated with neurodegenerative diseases, such as Alzheimer's, Parkinson's, and Amyotrophic lateral sclerosis (ALS), we determined their associated enriched disease pathways and the DEGs participating in each pathway (Fig. 11b). It's noteworthy that, we implied the differences among gene categories that trigger the various neurodegenerative disorders. In the case of the genes significantly associated with the Parkinson's and Alzheimer's diseases, they were mainly a part of the mitochondria, including the cytochrome c oxidase genes (cox), NDUF subunits of $\mathrm{NADH}$, and UQCRH gene families, that all are associated with the respiratory electron transport of mitochondria. The uniquely enriched ALS genes were more associated with the Mitogen-Activated protein 
kinase MAPK12, MAPK13, and MAPK14 and the TNF and TNF receptor superfamily genes (TNFRSF). On the other side, the IPA network analysis specifies a number of genes that play a role in central nervous system development, revealing a reduced gene expression for the brain-derived neurotrophic factor (BDNF) (Fig. S8). The results indicate that the influenza WSN virus can induce neuroinflammation and neurodegeneration pathways in hBMECs, which supports the hypothesis of previous literature that the influenza A virus induces symptoms like neurological diseases.

\section{Discussion}

This study provides considerable insights into the potential response of the hBMECs to the pathogenesis of the influenza A virus. The BBB disruption can be initiated by the cellular damage of brain endothelial cells, which could be followed by impaired brain homeostasis that induces an inflammatory immune response, neuronal cell death, and neurodegeneration (Sweeney, Sagare, and Zlokovic 2018; Erickson and Banks 2013; Parodi-Rullan, Sone, and Fossati 2019; Rumbaugh and Nath 2009; Stolp et al. 2013). Some preliminary work was carried out to study the influenza virus's influence on causing brain diseases (Jurgens, Amancherla, and Johnson 2012; Hosseini et al. 2018). However, we did not find enough answers about the impact of the influenza virus on the BBB precisely and whether it causes inevitable damage to the hBMECs. We used a neurotropic strain of influenza virus A/WSN/33 (H1N1) to infect the hBMECs (Mori et al. 1999). The WSN virus efficiently invaded the hBMECs, which has been noticed on the morphological basis by induced cell damage and molecular basis by the confirmed viral replication through RT-qPCR and protein expression through western blotting. Accordingly, the RNAseq at $12 \mathrm{hpi}$ revealed a set of DEGs with an increased level of immune-related and antiviral genes, including type I and III interferons, interferon-stimulated genes, proinflammatory cytokines, and chemokines such as IFN- $\beta$, IFN- $\lambda$, TNF, IFI44, ISG15, IL-6, CXCL2, CXCL3, CXCL8, CXCL10, CXCL11, and CXCL16. These results are consistent with previous research studying the influenza virus interaction with mouse cortical neurons that induced an increased level of the inflammatory cytokines, chemokines, and type I interferons (Wang et al. 2016).

The influenza virus NP translocates inside the cells by existing in the nucleus during early infection; later, it spreads into the cytoplasm and binds to the F-actin through specific residues (Neumann, Castrucci, and Kawaoka 1997; Zheng and Tao 2013; Digard et al. 1999). By using immunofluorescence, we noticed the accumulation of the virus NP in the hBMECs nucleus and scattered in the cytoplasm to form a colocalization with the actin cytoskeleton. Previous studies displayed that the actin network plays a major role in virus replication (Kumakura, Kawaguchi, and Nagata 2015; Gupta et al. 1998). The virus tends to be reduced by inhibiting the actin-myosin formation (Kumakura, Kawaguchi, and Nagata 2015). Additionally, a research study has indicated that cellular actin is necessary to activate human parainfluenza virus type 3 (HPIV3) transcription (Gupta et al. 1998). The infected hBMECs cells showed a morphological change in the actin cytoskeleton structure compared to intact cells (Fig. 2), coinciding with the activation of the gene set responsible for actin regulation (Fig. 5a-d). The genes activated could play a central role in intracellular transport, particle movement at the cell periphery before virus fusion, and the NP transcription, replication, and trafficking, such as the ACTC1 gene (König et al. 2010). Thus, we 
speculate that the influenza virus might enhance the cytoskeleton re-organization by increasing the Factin ratio (Lakadamyali et al. 2003). A similar conclusion reveals that the virus transport mechanism following endocytosis is mainly actin-dependent. That was further replaced by microtubules-based movement to the nucleus site where the viral RNA synthesis occurs, while NP and F-actin interaction was followed by cytoplasmic retention (Lakadamyali et al. 2003; Digard et al. 1999). Consistently, the cytoplasmic retention by NP might illustrate the reason behind the doughnut shape or the condensation of the F-actin towards the cell edges. The number of activated genes participating in the actin cytoskeleton organization was higher (185) than those suppressed the microtubule cytoskeleton organization (52) (Fig. 5a,c). Interestingly, the microtubules are known to mediate host responses to infection; they facilitate the antiretroviral activity of the TRIMs family(Elis, Ehrlich, and Bacharach 2015). Viruses can still evolve countermeasures to replicate successfully. For example, rabies virus P protein switches the host antiviral STAT1 from MT-facilitated to an MT-inhibited nuclear import process. Besides the virus's ability to block antiviral host responses, some viruses can also cause cellular-induced dysregulated cell division (Naghavi and Walsh 2017). Although most of the genes functioning in the cytoskeleton organization were upregulated following the IAV infection, a considerable number of genes were also suppressed. The exact roles played by the influenza virus on the microtubule organization are still under study; however, we predict from previous literature that the virus could induce the genes' suppression to fight against the antiviral host response.

The virus entrance can be detected by the activation of PRRs that included the activation of Toll-like receptors, RIG-I-like, and NOD-like receptors (Amarante-Mendes et al. 2018; Takeuchi and Akira 2010; Chen, Cheng, and Wang 2013). The antiviral genes are induced by the activation of the type I interferon signaling, which builds the first line of the host defence against the virus infection (Schneider, Chevillotte, and Rice 2014; McNab et al. 2015). The influenza virus likely induced the activation of PPRs in the hBMECs and activated the interferon signaling pathways to initiate a cascade of interferon-stimulated genes. Likewise, our results demonstrate a significant upregulation in the genes playing a crucial role in the cytokine-cytokine receptor interaction, JAK-STAT, neuroinflammation, and neuroactive ligand-receptor pathways. With slight differences from other findings discussing astrocytes' response to influenza A virus (Lin et al. 2015), hBMECs appear to be more susceptible to the virus infection than astrocytes. The neuroactive ligand-receptor interaction pathway was activated earlier in hBMECs at $12 \mathrm{hpi}$ than astrocytes at $24 \mathrm{hpi}$. In addition to a less induced downstream cascade of interferon signaling pathway at 24 hpi of astrocytes when compared to hBMECs at $12 \mathrm{hpi}$ (Lin et al. 2015),.

Rho GTPases are core regulatory molecules that link the surface receptors to organize actin and microtubule cytoskeleton (Bozza et al. 2015). On the other hand, we investigated that during the influenza A virus infection of hBMECs, there was an evident decline in the genes functioning in the RhoGDI signaling pathways. Thus, the ERM and Rho families cytoskeletal regulators showed an increased inhibition, while the F-actin was activated.

In line with previous studies, mitochondrial dysfunction is considered a primary reason for several neurological disorders (Wu, Chen, and Jiang 2019). Apoptosis is the principal pathological feature of 
neurodegeneration, which is controlled by the mitochondria (Wu, Chen, and Jiang 2019; Hroudová, Singh, and Fišar 2014). Our findings showed that the hBMECs infected with the influenza virus revealed several defects within the mitochondria, showing a pattern of inhibition within most of their biological processes, specifically in the electron transport chain, which plays a significant role in the pathogenesis of $A D$.

Few studies have examined the longer-term neurologic consequences due to influenza virus infection. Jang et al. have tracked the H5N1 virus once introduced to the mouse; they reported that the virus travels from the peripheral nervous system into the CNS to higher neuroaxis levels (Jang et al. 2009). They also indicated a significant loss of the dopaminergic neurons by $17 \%$ in the SNpc 60 days following H5N1 infection (Jang et al. 2009). Although the virus had disappeared from the brain in 21 days, there was a long-lasting microglial activation (Jang et al. 2009). Moreover, a group of researchers specified more information about the long-term consequences for the CNS infection with neurotropic and nonneurotropic Influenza A virus (IAV) strains (Hosseini et al. 2018). They outlined a significant spine loss in the hippocampus and a microglia activation during the acute phase of the disease with the neurotropic H7N7 and the non-neurotropic H3N2 in mice (Hosseini et al. 2018). Both neurotropic and non-neurotropic strains indicated a significant reduction in spine number 30 days post-infection. Complete recovery was noticed at $120 \mathrm{dpi}$, which provides evidence for long-term disturbances in the CNS (Hosseini et al. 2018).

With casting a light on the previous research, we conclude that s neurotropic viruses such as the human immunodeficiency virus (HIV), and the Japanese encephalitis virus could invade the CNS and cause diseases. We are currently more curious about how some viruses could spread from the respiratory system to the CNS and induce diseases. It was not only for the influenza viruses but also the syncytial virus (hRSV), the human metapneumovirus (hMPV), and coronavirus (CoV), that were all detected in the cerebrospinal fluid (CFS) following infection (Bohmwald et al. 2018). The hRSV, which induces a respiratory illness in infants, also alters the neurologic homeostasis with seizures, ataxia, and other encephalopathy symptoms (Espinoza et al. 2013). It translocates from the lung to invade the CNS through a hematogenous/blood-brain barrier route and releases humoral neurotoxic cytokine mediators (Park and Suh 2014). The hMPV respiratory pathogen severely infects newborns' respiratory systems and immunocompromised individuals (Shafagati and Williams 2018; Edwards et al. 2013). During the last two decades, hMPV was observed with a potential neuroinvasion. They reported different cases of febrile seizures, encephalitis, and encephalopathies parallel to the existence of the virus RNA in the CSF (Jeannet et al. 2017; Sánchez Fernández et al. 2012; Desforges et al. 2019). In a similar pattern to the influenza virus, the HCoV could enter the CNS through the olfactory bulb upon nasal infection (Arbour et al. 2000). Neuroinvasion was noticed along with the presence of the HCoV RNA in brains, as it was detected in the human brain parenchyma of patients with multiple sclerosis (Arbour et al. 2000; Burks et al. 1980). Likewise, the primary glial culture, when infected with HCoV secretes TNF-a, IL-12p40, IL-15, IL6, CXCL9, and CXCL10 (Bohmwald et al. 2018). The recent coronavirus, SARS-CoV-2 utilizes the angiotensin-converting enzyme 2 (ACE2) receptor to get into the cells (Baig et al. 2020). It can attack the CNS by targeting the ACE2 receptors expressed on the neurons, glial tissues, and brain vasculature, as it was further associated with neurological manifestations (Turner, Hiscox, and Hooper 2004; Kabbani and Olds 2020; Ahmed et al. 2020).

Page 13/36 
To conclude, neurodegenerative disorders such as AD, Parkinson's, and ALS, usually observed in elderly persons, are characterized by neuronal cell death. They could be activated following virus attacks by several inflammatory processes (Chen, Zhang, and Huang 2016). As long as neuronal cell death is not regenerated, we speculate that successive infection with the influenza virus might play a potential role in the appearance of neuronal disorders over time. It is also essential to realize that understanding the underlying interaction between neuronal and inflammatory immune cells could solve a future problem for the various neurodegenerative disorders. For example, senile plaques and neurofibrillary tangles of $A D$ could induce a route of neuroinflammation that triggers the pathogenesis of the disease as much as or even more than the plaques themselves (Zhang et al. 2013; Heneka et al. 2015). AD pathogenesis progression occurs after a robust immunological interaction. The misfolded protein bind to the PRRs on astroglia and microglia and further induce innate immune and proinflammatory response(Heneka et al. 2015). The incidence of symptoms like neurodegenerative disease or the activation of their related pathways following infection with the influenza A virus should be critical for answering the upcoming research questions regarding the induction of $A D$ and other neurodegenerative disorders in the long run. Moreover, it might also be a useful model of study for future therapeutics production and neurodegenerative disease prevention.

\section{Conclusions}

Our results demonstrate the susceptibility of hBMECs as a part of the BBB to become infected by WSN. The infection was followed by robust innate immune and inflammatory signaling activation, disruption in the cell morphology and cytoskeletal structure, dysfunction on the mitochondrial level, and activation of neuroglia and neurodegenerative disease pathways.

\section{Declarations}

\section{Ethics approval and consent to participate}

\section{Consent for publication}

Not Applicable.

\section{Availability of data and materials}

Data deposition: The sequence reported to this article for the hBMECs experiment has been deposited to the NCBI Sequence Read Archive (SRA) under the accession no. (PRJNA615331).

\section{Competing interests}

The authors declare that they have no competing interests.

\section{Funding}


The authors are grateful for the financial support provided by the National Program on Key Research Project of China (2016YFD0500406), the National Natural Sciences Foundation of China (Grant No. 31872455), the Fundamental Research Funds for the Central Universities (2662018PY016), and the Start-up Research Fund from Huazhong Agricultural University.

\section{Authors' contributions}

$\mathrm{MC}$ and $\mathrm{DH}$ managed the experimental design and research question. DH performed the wet lab work for hBMECs related experiments. XG preserved and expanded the A/WSN/33 influenza virus strain. WK has aided in cell culture work. XL prepared the RNA samples ready for sequencing from hBMECs. TX and DH have done the mapping and assembly of RNAseq raw data. DH has done the annotation and designed the output figures. DH and MC wrote and edited the manuscript.

\section{Acknowledgments}

The authors thank Ms. Wenjing Xiong for her technical assistance.

\section{Affiliations}

${ }^{1}$ State Key Laboratory of Agricultural Microbiology, College of Veterinary Medicine, Huazhong Agricultural University, Wuhan 430070, Hubei, China

Doaa Higazy, Xianwu Lin, Ke Wang, Xiaochen Gao, Min Cui

${ }^{2}$ Key Laboratory of Preventive Veterinary Medicine in Hubei Province, The Cooperative Innovation Center for Sustainable Pig Production, Wuhan 430070, Hubei, China

Doaa Higazy, Xianwu Lin, Ke Wang, Xiaochen Gao, Min Cui

${ }^{3}$ Key Laboratory of Development of Veterinary Diagnostic Products, Ministry of Agriculture of the People's Republic of China, Wuhan 430070, Hubei, China

Doaa Higazy, Xianwu Lin, Ke Wang, Xiaochen Gao, Min Cui

${ }^{4}$ International Research Center for Animal Disease, Ministry of Science and Technology of the People's Republic of China, Wuhan 430070, Hubei, China

Doaa Higazy, Xianwu Lin, Ke Wang, Xiaochen Gao, Min Cui

${ }^{5}$ College of Informatics, Huazhong Agricultural University, Wuhan 430070, Hubei, China

Tanghui Xie

${ }^{6}$ Microbiology Department, Faculty of Agriculture, Cairo University, 12613 Giza, Egypt. 
Doaa Higazy

\section{Abbreviations}

BBB

Blood-Brain Barrier

CNS

Central nervous system

hBMECs

human brain microvascular endothelial cells

DEGs

Differentially expressed genes

GO

gene ontology

KEGG

Kyoto Encyclopedia of Genes and Genomes

IAV

Influenza A virus

BDNF

Brain-derived neurotrophic factor

NGF

Nerve growth factor

SNpc

Substantia nigra pars compacta

MOI

Multiplicity of infection

PCR

Polymerase chain reaction

ORA

Over Representation Analysis

GSEA

Gene set enrichment analysis

IFITs

an interferon-induced protein with tetratricopeptide repeats

ISGs

Interferon stimulating genes

IFIs

Interferon-induced proteins

IPA 
Ingenuity Pathway Analysis

PRRs

Pattern recognition receptors

FBS

fetal bovine serum

MDCK

Madin-Darby Canine Kidney

PBS

Phosphate-buffered saline

DNB

DNA nano ball

RIPA

Radioimmunoprecipitation assay

CSF

Cerebrospinal fluid

\section{References}

Ahmed, Muhammad Umer, Muhammad Hanif, Mukarram Jamat Ali, Muhammad Adnan Haider, Danish Kherani, Gul Muhammad Memon, Amin H. Karim, and Abdul Sattar. 2020. 'Neurological Manifestations of COVID-19 (SARS-CoV-2): A Review', 11.

Amarante-Mendes, Gustavo P., Sandy Adjemian, Laura Migliari Branco, Larissa C. Zanetti, Ricardo Weinlich, and Karina R. Bortoluci. 2018. 'Pattern Recognition Receptors and the Host Cell Death Molecular Machinery', Frontiers in Immunology, 9.

Anthony, D. C., Y. Couch, P. Losey, and M. C. Evans. 2012. 'The systemic response to brain injury and disease', Brain Behav Immun, 26: 534-40.

Arbour, N., R. Day, J. Newcombe, and P. J. Talbot. 2000. 'Neuroinvasion by human respiratory coronaviruses', J Virol, 74: 8913-21.

Aronsson, F., H. Karlsson, H. G. Ljunggren, and K. Kristensson. 2001. 'Persistence of the influenza A/WSN/33 virus RNA at midbrain levels of immunodefective mice', J Neurovirol, 7: 117-24.

Baig, Abdul Mannan, Areeba Khaleeq, Usman Ali, and Hira Syeda. 2020. 'Evidence of the COVID-19 Virus Targeting the CNS: Tissue Distribution, Host-Virus Interaction, and Proposed Neurotropic Mechanisms', ACS Chemical Neuroscience, 11: 995-98.

Barbosa-Silva, M. C., L. E. Santos, and B. Rangel. 2018. 'The Impact of Non-Neurotropic Influenza Strains on the Brain: A Role for Microglial Priming?', J Neurosci, 38: 7758-60. 
Billa, R. D., T. Czech, A. Badheka, and M. Chegondi. 2020. 'Influenza B associated acute necrotising encephalopathy with visual impairment in a child', BMJ Case Rep, 13.

Blackmore, S., J. Hernandez, M. Juda, E. Ryder, G. G. Freund, R. W. Johnson, and A. J. Steelman. 2017. 'Influenza infection triggers disease in a genetic model of experimental autoimmune encephalomyelitis', Proc Natl Acad Sci U S A, 114: E6107-E16.

Blighe K, Rana S, Lewis M. 2020. 'EnhancedVolcano: Publication-ready volcano plots with enhanced colouring and labeling'.

Bohmwald, Karen, Nicolás M. S. Gálvez, Mariana Ríos, and Alexis M. Kalergis. 2018. 'Neurologic Alterations Due to Respiratory Virus Infections', Frontiers in cellular neuroscience, 12: 386-86.

Bozza, William P., Yaqin Zhang, Kory Hallett, Leslie A. Rivera Rosado, and Baolin Zhang. 2015. 'RhoGDI deficiency induces constitutive activation of Rho GTPases and COX-2 pathways in association with breast cancer progression', Oncotarget, 6: 32723-36.

Breuer, K., A. K. Foroushani, M. R. Laird, C. Chen, A. Sribnaia, R. Lo, G. L. Winsor, R. E. Hancock, F. S. Brinkman, and D. J. Lynn. 2013. 'InnateDB: systems biology of innate immunity and beyond-recent updates and continuing curation', Nucleic Acids Res, 41: D1228-33.

Burks, J. S., B. L. DeVald, L. D. Jankovsky, and J. C. Gerdes. 1980. 'Two coronaviruses isolated from central nervous system tissue of two multiple sclerosis patients', Science, 209: 933-4.

Chaves, A. J., J. Vergara-Alert, N. Busquets, R. Valle, R. Rivas, A. Ramis, A. Darji, and N. Majo. 2014. 'Neuroinvasion of the highly pathogenic influenza virus H7N1 is caused by disruption of the blood brain barrier in an avian model', PLOS ONE, 9: e115138.

Chen, Shun, Anchun Cheng, and Mingshu Wang. 2013. 'Innate sensing of viruses by pattern recognition receptors in birds', Veterinary Research, 44: 82.

Chen, W. W., X. Zhang, and W. J. Huang. 2016. 'Role of neuroinflammation in neurodegenerative diseases (Review)', Mol Med Rep, 13: 3391-6.

Daneman, R., and A. Prat. 2015. 'The blood-brain barrier', Cold Spring Harb Perspect Biol, 7: a020412.

Desforges, M., A. Le Coupanec, P. Dubeau, A. Bourgouin, L. Lajoie, M. Dubé, and P. J. Talbot. 2019. 'Human Coronaviruses and Other Respiratory Viruses: Underestimated Opportunistic Pathogens of the Central Nervous System?', Viruses, 12.

Digard, Paul, Debra Elton, Konrad Bishop, Elizabeth Medcalf, Alan Weeds, and Brian Pope. 1999. 'Modulation of Nuclear Localization of the Influenza Virus Nucleoprotein through Interaction with Actin Filaments', Journal of Virology, 73: 2222-31. 
Edwards, K. M., Y. Zhu, M. R. Griffin, G. A. Weinberg, C. B. Hall, P. G. Szilagyi, M. A. Staat, M. Iwane, M. M. Prill, and J. V. Williams. 2013. 'Burden of human metapneumovirus infection in young children', $N$ Engl J Med, 368: 633-43.

Elis, Efrat, Marcelo Ehrlich, and Eran Bacharach. 2015. 'Dynamics and restriction of murine leukemia virus cores in mitotic and interphase cells', Retrovirology, 12: 95.

Erickson, M. A., and W. A. Banks. 2013. 'Blood-brain barrier dysfunction as a cause and consequence of Alzheimer's disease', J Cereb Blood Flow Metab, 33: 1500-13.

Espinoza, Janyra A., Karen Bohmwald, Pablo F. Céspedes, Roberto S. Gómez, Sebastián A. Riquelme, Claudia M. Cortés, Javier A. Valenzuela, Rodrigo A. Sandoval, Floria C. Pancetti, Susan M. Bueno, Claudia A. Riedel, and Alexis M. Kalergis. 2013. 'Impaired learning resulting from respiratory syncytial virus infection', Proceedings of the National Academy of Sciences of the United States of America, 110: 911217.

Frensing, Timo, Sascha Y. Kupke, Mandy Bachmann, Susanne Fritzsche, Lili E. Gallo-Ramirez, and Udo Reichl. 2016. 'Influenza virus intracellular replication dynamics, release kinetics, and particle morphology during propagation in MDCK cells', Applied microbiology and biotechnology, 100: 7181-92.

Garcia-Monco, J. C., I. E. Cortina, E. Ferreira, A. Martínez, L. Ruiz, A. Cabrera, and M. G. Beldarrain. 2011. 'Reversible splenial lesion syndrome (RESLES): what's in a name?', J Neuroimaging, 21: e1-14.

Gupta, Sanhita, Bishnu P. De, Judith A. Drazba, and Amiya K. Banerjee. 1998. 'Involvement of Actin Microfilaments in the Replication of Human Parainfluenza Virus Type 3', Journal of Virology, 72: 265562.

Heneka, M. T., M. J. Carson, J. El Khoury, G. E. Landreth, F. Brosseron, D. L. Feinstein, A. H. Jacobs, T. Wyss-Coray, J. Vitorica, R. M. Ransohoff, K. Herrup, S. A. Frautschy, B. Finsen, G. C. Brown, A. Verkhratsky, K. Yamanaka, J. Koistinaho, E. Latz, A. Halle, G. C. Petzold, T. Town, D. Morgan, M. L. Shinohara, V. H. Perry, C. Holmes, N. G. Bazan, D. J. Brooks, S. Hunot, B. Joseph, N. Deigendesch, O. Garaschuk, E. Boddeke, C. A. Dinarello, J. C. Breitner, G. M. Cole, D. T. Golenbock, and M. P. Kummer. 2015. 'Neuroinflammation in Alzheimer's disease', Lancet Neurol, 14: 388-405.

Hosseini, S., E. Wilk, K. Michaelsen-Preusse, I. Gerhauser, W. Baumgartner, R. Geffers, K. Schughart, and M. Korte. 2018. 'Long-Term Neuroinflammation Induced by Influenza A Virus Infection and the Impact on Hippocampal Neuron Morphology and Function', J Neurosci, 38: 3060-80.

Hroudová, Jana, Namrata Singh, and Zdeněk Fišar. 2014. 'Mitochondrial Dysfunctions in Neurodegenerative Diseases: Relevance to Alzheimer's Disease', BioMed Research International, 2014: 175062. 
Jang, H., D. Boltz, J. McClaren, A. K. Pani, M. Smeyne, A. Korff, R. Webster, and R. J. Smeyne. 2012. 'Inflammatory effects of highly pathogenic H5N1 influenza virus infection in the CNS of mice', J Neurosci, 32: $1545-59$.

Jang, H., D. Boltz, K. Sturm-Ramirez, K. R. Shepherd, Y. Jiao, R. Webster, and R. J. Smeyne. 2009. 'Highly pathogenic H5N1 influenza virus can enter the central nervous system and induce neuroinflammation and neurodegeneration', Proc Natl Acad Sci U S A, 106: 14063-8.

Jeannet, N., B. G. van den Hoogen, J. C. Schefold, F. Suter-Riniker, and R. Sommerstein. 2017. 'Cerebrospinal Fluid Findings in an Adult with Human Metapneumovirus-Associated Encephalitis', Emerg Infect Dis, 23: 370.

Jurgens, Heidi A., Kaushik Amancherla, and Rodney W. Johnson. 2012. 'Influenza Infection Induces Neuroinflammation, Alters Hippocampal Neuron Morphology, and Impairs Cognition in Adult Mice', The Journal of Neuroscience, 32: 3958-68.

Kabbani, N., and J. L. Olds. 2020. 'Does COVID19 Infect the Brain? If So, Smokers Might Be at a Higher Risk', Mol Pharmacol, 97: 351-53.

Kim, Daehwan, Ben Langmead, and Steven L. Salzberg. 2015. 'HISAT: a fast spliced aligner with low memory requirements', Nature Methods, 12: 357-60.

König, Renate, Silke Stertz, Yingyao Zhou, Atsushi Inoue, H. Heinrich Hoffmann, Suchita Bhattacharyya, Judith G. Alamares, Donna M. Tscherne, Mila B. Ortigoza, Yuhong Liang, Qinshan Gao, Shane E. Andrews, Sourav Bandyopadhyay, Paul De Jesus, Buu P. Tu, Lars Pache, Crystal Shih, Anthony Orth, Ghislain Bonamy, Loren Miraglia, Trey Ideker, Adolfo García-Sastre, John A. T. Young, Peter Palese, Megan L. Shaw, and Sumit K. Chanda. 2010. 'Human host factors required for influenza virus replication', Nature, 463: 813 $-17$.

Koyuncu, O. O., I. B. Hogue, and L. W. Enquist. 2013. 'Virus infections in the nervous system', Cell Host Microbe, 13: 379-93.

Kramer, A, J Green, and J Pollard Jr. 2014. 'Tugendreich S. Causal analysis approaches in Ingenuity Pathway Analysis', Bioinformatics, 30: 523 - 30.

Kumakura, Michiko, Atsushi Kawaguchi, and Kyosuke Nagata. 2015. 'Actin-myosin network is required for proper assembly of influenza virus particles', Virology, 476: $141-50$.

Lakadamyali, Melike, Michael J. Rust, Hazen P. Babcock, and Xiaowei Zhuang. 2003. 'Visualizing infection of individual influenza viruses', Proceedings of the National Academy of Sciences, 100: 9280-85.

Lampa, Jon, Marie Westman, Diana Kadetoff, Anna Nordenstedt Agréus, Erwan Le Maître, Caroline GillisHaegerstrand, Magnus Andersson, Mohsen Khademi, Maripat Corr, Christina A. Christianson, Ada Delaney, Tony L. Yaksh, Eva Kosek, and Camilla I. Svensson. 2012. 'Peripheral inflammatory disease 
associated with centrally activated IL-1 system in humans and mice', Proceedings of the National Academy of Sciences, 109: 12728-33.

Lin, X., R. Wang, J. Zhang, X. Sun, Z. Zou, S. Wang, and M. Jin. 2015. 'Insights into Human Astrocyte Response to H5N1 Infection by Microarray Analysis', Viruses, 7: 2618-40.

Love, M.I., Huber, W., Anders, S. 2014. 'Moderated estimation of fold change and dispersion for RNA-seq data with DESeq2', Genome Biology, 15: 550.

McNab, Finlay, Katrin Mayer-Barber, Alan Sher, Andreas Wack, and Anne O'Garra. 2015. 'Type I interferons in infectious disease', Nature Reviews Immunology, 15: 87-103.

Mori, I., A. D. Diehl, A. Chauhan, H. G. Ljunggren, and K. Kristensson. 1999. 'Selective targeting of habenular, thalamic midline and monoaminergic brainstem neurons by neurotropic influenza A virus in mice', J Neurovirol, 5: 355-62.

Naghavi, Mojgan H., and Derek Walsh. 2017. 'Microtubule Regulation and Function during Virus Infection', Journal of Virology, 91: e00538-17.

Nassif, X., S. Bourdoulous, E. Eugène, and P. O. Couraud. 2002. 'How do extracellular pathogens cross the blood-brain barrier?', Trends Microbiol, 10: 227-32.

Neumann, G., M. R. Castrucci, and Y. Kawaoka. 1997. 'Nuclear import and export of influenza virus nucleoprotein', Journal of Virology, 71: 9690-700.

Park, Arim, and Sangil Suh. 2014. 'Respiratory syncytial virus-related encephalitis: magnetic resonance imaging findings with diffusion-weighted study: response to a "letter to the editor"', Neuroradiology, 56: 433.

Park, C. H., M. Ishinaka, A. Takada, H. Kida, T. Kimura, K. Ochiai, and T. Umemura. 2002. 'The invasion routes of neurovirulent A/Hong Kong/483/97 (H5N1) influenza virus into the central nervous system after respiratory infection in mice', Arch Virol, 147: 1425-36.

Parodi-Rullan, R., J. Y. Sone, and S. Fossati. 2019. 'Endothelial Mitochondrial Dysfunction in Cerebral Amyloid Angiopathy and Alzheimer's Disease', J Alzheimers Dis, 72: 1019-39.

Portela, Agustín, and Paul Digard. 2002. 'The influenza virus nucleoprotein: a multifunctional RNA-binding protein pivotal to virus replication', Journal of General Virology, 83: 723-34.

Rohn, T. T., and L. W. Catlin. 2011. 'Immunolocalization of influenza A virus and markers of inflammation in the human Parkinson's disease brain', PLOS ONE, 6: e20495.

Rosas-Hernandez, H., E. Cuevas, S. M. Lantz, M. G. Paule, and S. F. Ali. 2018. 'Isolation and Culture of Brain Microvascular Endothelial Cells for In Vitro Blood-Brain Barrier Studies', Methods Mol Biol, 1727: 
Rüdiger, Daniel, Sascha Young Kupke, Tanja Laske, Pawel Zmora, and Udo Reichl. 2019. 'Multiscale modeling of influenza A virus replication in cell cultures predicts infection dynamics for highly different infection conditions', PLOS Computational Biology, 15: e1006819.

Rumbaugh, Jeffrey A., and Avindra Nath. 2009. 'Neuronal Cell Death and Inflammation.' in Marc D. Binder, Nobutaka Hirokawa and Uwe Windhorst (eds.), Encyclopedia of Neuroscience (Springer Berlin Heidelberg: Berlin, Heidelberg).

Sánchez Fernández, Iván, Mónica Rebollo Polo, Carmen Muñoz-Almagro, Laura Monfort Carretero, Sergio Fernández Ureña, Adela Rueda Muñoz, Roser Colomé Roura, and Belén Pérez Dueñas. 2012. 'Human Metapneumovirus in the Cerebrospinal Fluid of a Patient With Acute Encephalitis', Archives of Neurology, 69: $649-52$.

Schneider, W. M., M. D. Chevillotte, and C. M. Rice. 2014. 'Interferon-stimulated genes: a complex web of host defenses', Annual review of immunology, 32: 513-45.

Shafagati, Nazly, and John Williams. 2018. 'Human metapneumovirus - what we know now', F1000Research, 7: $135-35$.

Stins, M. F., F. Gilles, and K. S. Kim. 1997. 'Selective expression of adhesion molecules on human brain microvascular endothelial cells', J Neuroimmunol, 76: 81-90.

Stins, Monique F., Julie Badger, and Kwang Sik Kim. 2001. 'Bacterial invasion and transcytosis in transfected human brain microvascular endothelial cells', Microbial Pathogenesis, 30: 19-28.

Stolp, H. B., S. A. Liddelow, I. Sa-Pereira, K. M. Dziegielewska, and N. R. Saunders. 2013. 'Immune responses at brain barriers and implications for brain development and neurological function in later life', Front Integr Neurosci, 7: 61.

Studahl, M. 2003. 'Influenza virus and CNS manifestations', J Clin Virol, 28: 225-32.

Sweeney, M. D., A. P. Sagare, and B. V. Zlokovic. 2018. 'Blood-brain barrier breakdown in Alzheimer disease and other neurodegenerative disorders', Nat Rev Neurol, 14: 133-50.

Takeuchi, O., and S. Akira. 2010. 'Pattern recognition receptors and inflammation', Cell, 140: 805 - 20.

Terrier, O., C. Carron, B. De Chassey, J. Dubois, A. Traversier, T. Julien, G. Cartet, A. Proust, S. Hacot, D. Ressnikoff, V. Lotteau, B. Lina, J. J. Diaz, V. Moules, and M. Rosa-Calatrava. 2016. 'Nucleolin interacts with influenza A nucleoprotein and contributes to viral ribonucleoprotein complexes nuclear trafficking and efficient influenza viral replication', Sci Rep, 6: 29006. 
Tesoriero, C., A. Codita, M. D. Zhang, A. Cherninsky, H. Karlsson, G. Grassi-Zucconi, G. Bertini, T. Harkany, K. Ljungberg, P. Liljestrom, T. G. Hokfelt, M. Bentivoglio, and K. Kristensson. 2016. 'H1N1 influenza virus induces narcolepsy-like sleep disruption and targets sleep-wake regulatory neurons in mice', Proc Natl Acad Sci U S A, 113: E368-77.

Turner, A. J., J. A. Hiscox, and N. M. Hooper. 2004. 'ACE2: from vasopeptidase to SARS virus receptor', Trends Pharmacol Sci, 25: 291-4.

van Riel, D., L. M. Leijten, R. M. Verdijk, C. GeurtsvanKessel, E. van der Vries, A. M. van Rossum, A. D. Osterhaus, and T. Kuiken. 2014. 'Evidence for influenza virus CNS invasion along the olfactory route in an immunocompromised infant', J Infect Dis, 210: 419-23.

Vanderschueren, G., K. Schotsmans, E. Maréchal, and R. Crols. 2018. 'Mild encephalitis with reversible splenial (MERS) lesion syndrome due to influenza B virus', Pract Neurol, 18: 391-92.

Ventresca, Silvia, Claudia Guiducci, Sara Tagliani, Sara Dal Bo, Paolo Ricciardelli, Patrizia Cenni, and Federico Marchetti. 2021. 'Clinically Mild Encephalopathy with a Reversible Splenial Lesion Caused by Influenza B Virus in an Unvaccinated Child', Pediatric reports, 13: 72-75.

Wang, G., R. Li, Z. Jiang, L. Gu, Y. Chen, J. Dai, and K. Li. 2016. 'Influenza Virus Induces Inflammatory Response in Mouse Primary Cortical Neurons with Limited Viral Replication', Biomed Res Int, 2016: 8076989.

Wickham, Hadley. 2009. ggplot2: elegant graphics for data analysis (Springer New York).

Wu, Y., M. Chen, and J. Jiang. 2019. 'Mitochondrial dysfunction in neurodegenerative diseases and drug targets via apoptotic signaling', Mitochondrion, 49: 35-45.

Yang, R., W. Liu, L. Miao, X. Yang, J. Fu, B. Dou, A. Cai, X. Zong, C. Tan, H. Chen, and X. Wang. 2016. 'Induction of VEGFA and Snail-1 by meningitic Escherichia coli mediates disruption of the blood-brain barrier', Oncotarget, 7: 63839-55.

Yu, G., L. G. Wang, Y. Han, and Q. Y. He. 2012. 'clusterProfiler: an R package for comparing biological themes among gene clusters', Omics, 16: 284-7.

Yu, G., L. G. Wang, G. R. Yan, and Q. Y. He. 2015. 'DOSE: an R/Bioconductor package for disease ontology semantic and enrichment analysis', Bioinformatics, 31: 608-9.

Yu, Guangchuang. 2019. 'enrichplot: Visualization of Functional Enrichment Result'.

Zhang, B., C. Gaiteri, L. G. Bodea, Z. Wang, J. McElwee, A. A. Podtelezhnikov, C. Zhang, T. Xie, L. Tran, R. Dobrin, E. Fluder, B. Clurman, S. Melquist, M. Narayanan, C. Suver, H. Shah, M. Mahajan, T. Gillis, J. Mysore, M. E. MacDonald, J. R. Lamb, D. A. Bennett, C. Molony, D. J. Stone, V. Gudnason, A. J. Myers, E. E. 
Schadt, H. Neumann, J. Zhu, and V. Emilsson. 2013. 'Integrated systems approach identifies genetic nodes and networks in late-onset Alzheimer's disease', Cell, 153: 707 - 20.

Zheng, Wenjie, and Yizhi Jane Tao. 2013. 'Structure and assembly of the influenza A virus ribonucleoprotein complex', FEBS Letters, 587: 1206-14.

Zhu, A., J. G. Ibrahim, and M. I. Love. 2019. 'Heavy-tailed prior distributions for sequence count data: removing the noise and preserving large differences', Bioinformatics, 35: 2084-92.

\section{Figures}

a
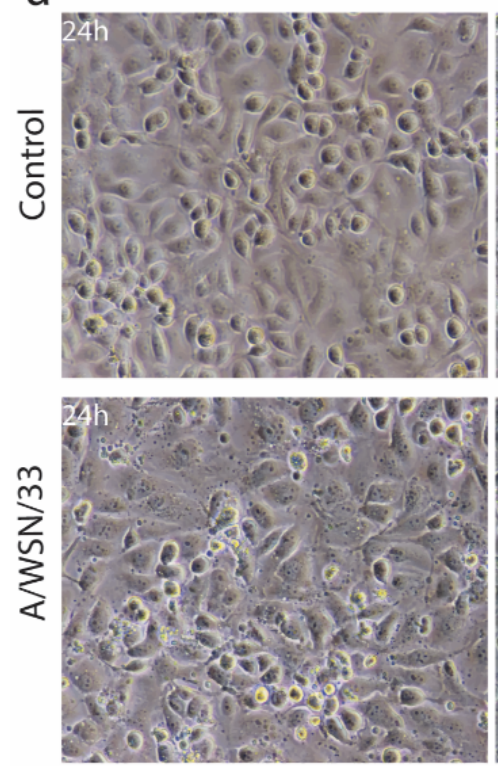

$\mathrm{b}$
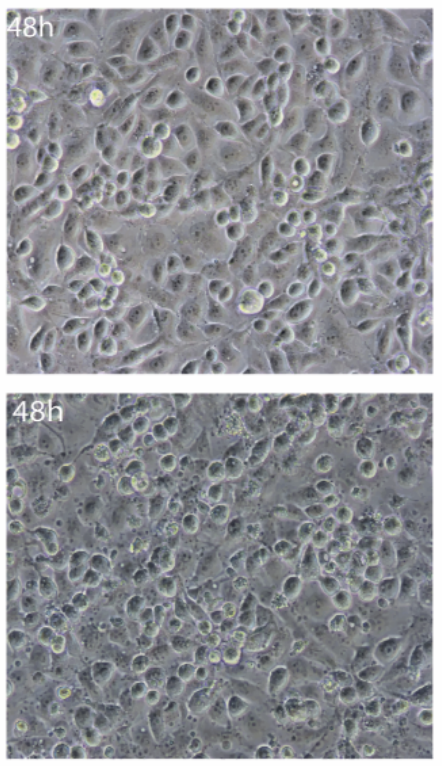

C
NP (A/WSN/33)
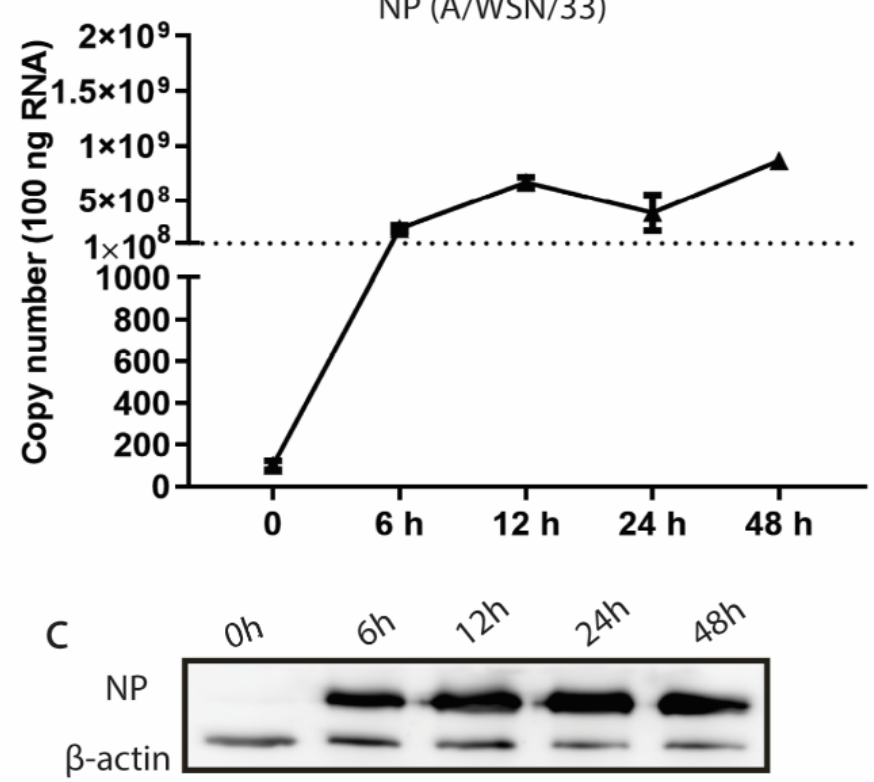

Figure 1

Influenza virus entry and infection to hBMECs. a Phase-contrast (Ph) microscopy visualization of hBMECs morphology infected by A/WSN/33 (H1N1) at $0.1 \mathrm{MOI}$. b RNA extracted from hBMECs and targeted by RT-qPCR to detect NP gene expression at different time points, error bars indicate standard deviation. $\mathrm{c}$ the cells were lysed for NP detection by western blotting at indicated time points postinfection. Data were shown as means \pm SEM from three experiments, and statistical significance analyzed by t-test $* p<0.05, * \star *<0.001$. 


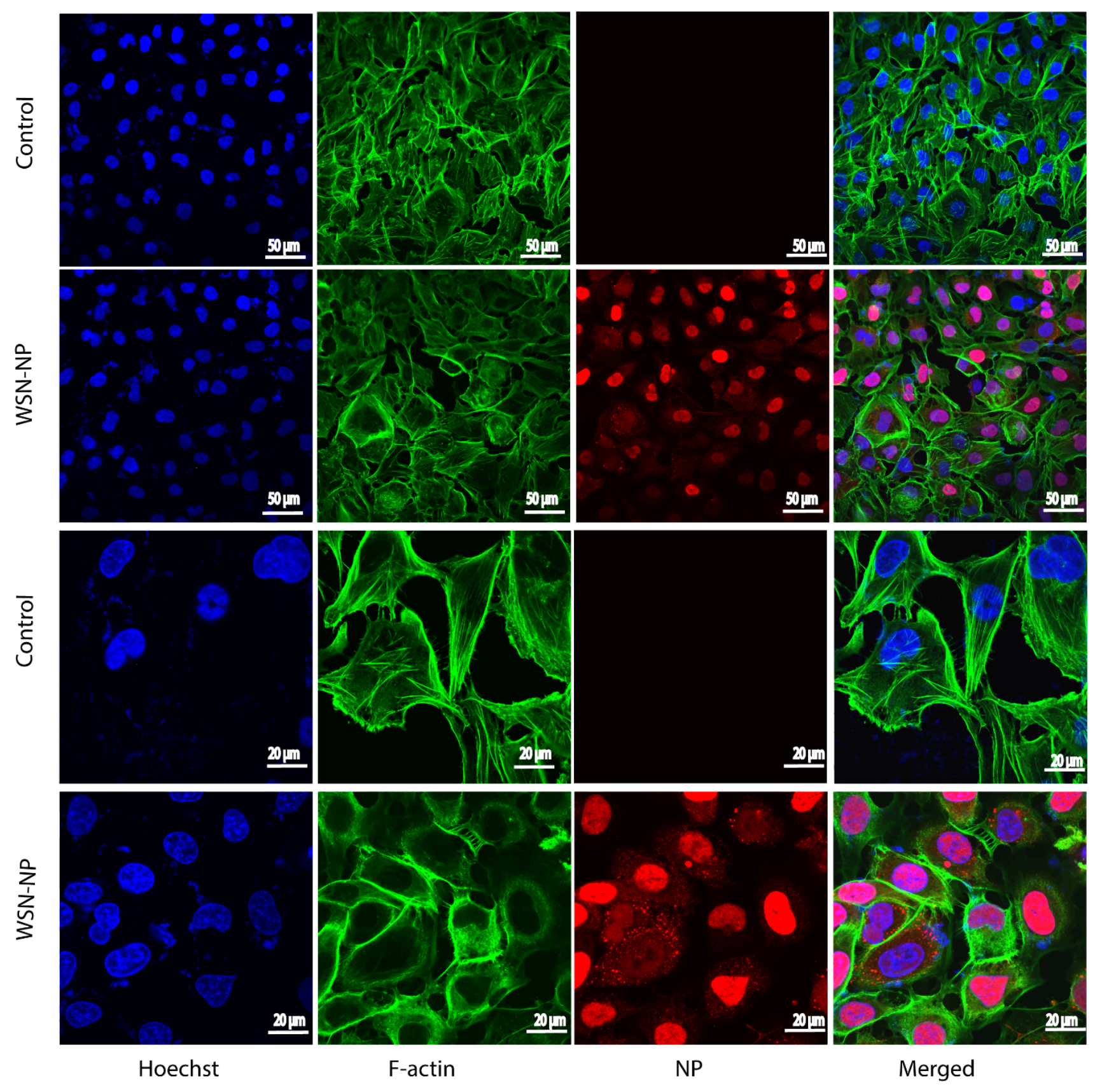

\section{Figure 2}

Immunoflourence for hBMECs at $12 \mathrm{hpi}$ with influenza A virus. hBMECs were mock-treated or infected with A/WSN/33 (H1N1) virus at $0.1 \mathrm{MOI}$. After $12 \mathrm{~h}$, the cells were stained with anti-NP (red), Phalloidin for F-actin (green), and Hoechst for nucleic acid stain (Blue). Images were captured by confocal microscopy. The column "Merged" is generated by the machine software, which is produced by 
positioning the "red", "green" and "blue" fluorescence of the same cells within the same optical plane: scale bar $50,20 \mu \mathrm{M}$.

a

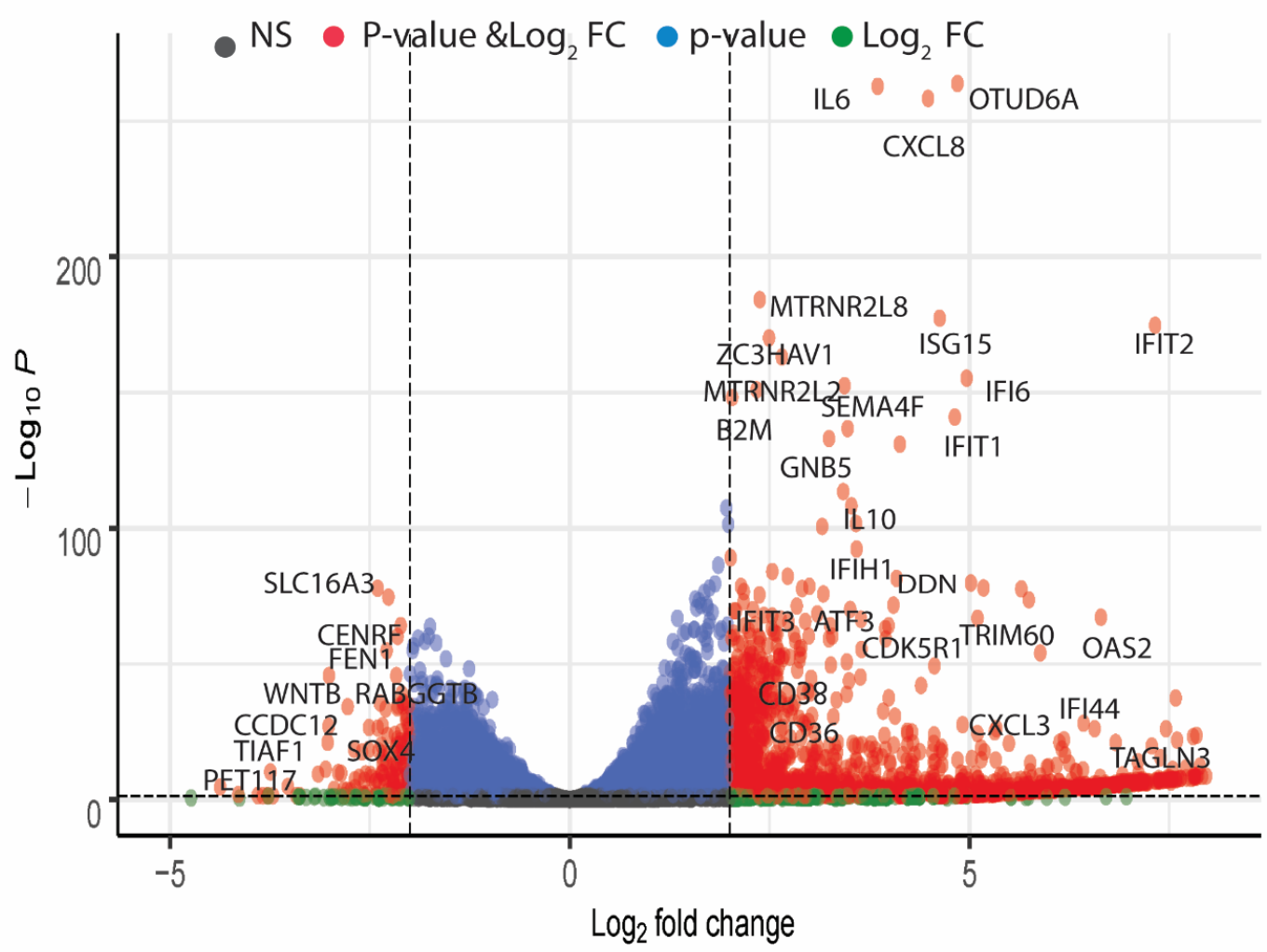

b
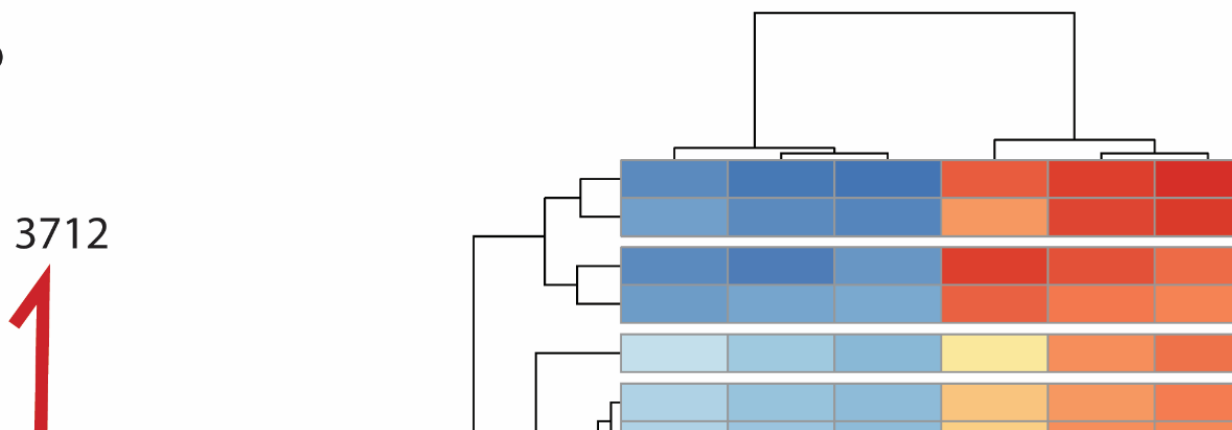

5500
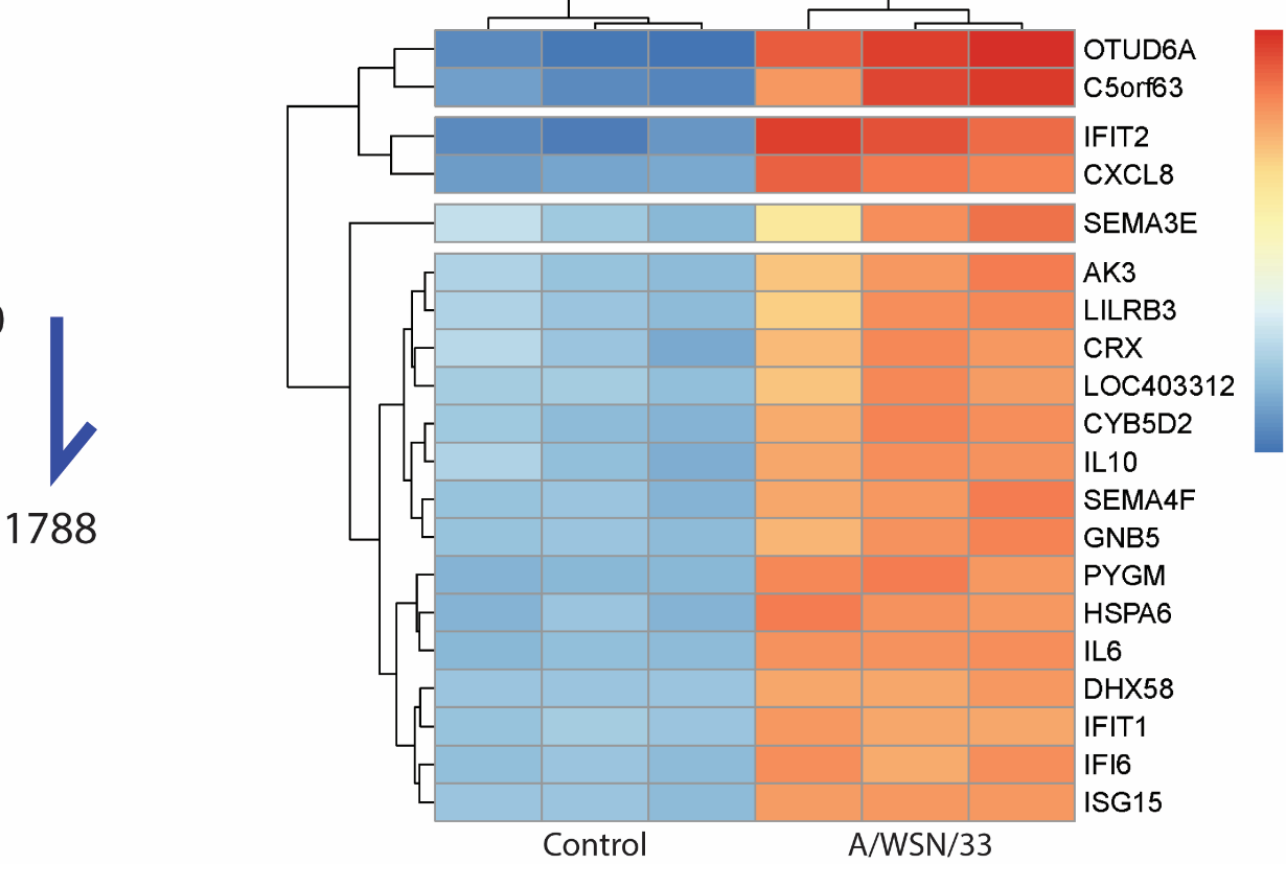

Figure 3

Transcriptomic analysis of hBMECs following infection with influenza A virus. a Volcano plot of host genes differentially expressed at $12 \mathrm{hpi} \mathrm{(padj<0.05)}$ and \pm 2 log2FC change. Each dot represents a gene. The red, blue, green, and black dots represent the differentially expressed genes within the selected p- 
value (padj< 0.05 ) and log $2 F C \pm 2$, the $p$-value, the log2FC, and non-significant genes, respectively. $b$ Heatmap of the top 20 genes induced in hBMECs at $12 \mathrm{hpi}$ (padj< 0.05 ). A list of the differentially expressed genes is available in Supplementary file 2.

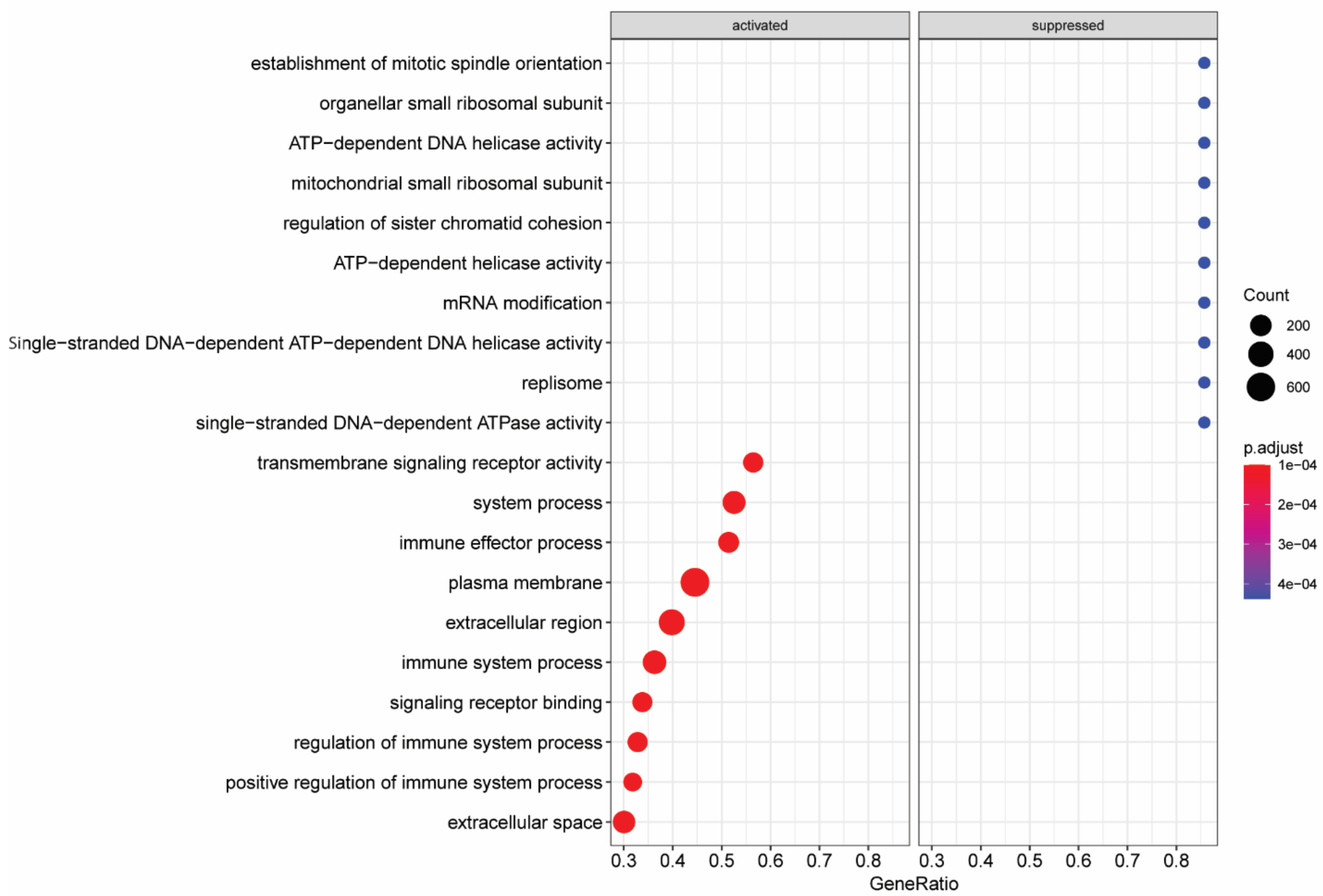

\section{Figure 4}

Functional enrichment analysis of the DEGs in hBMECs at $12 \mathrm{hpi}$. Gene ontology terms plotted in the order of gene ratio, the size of the dots depicts the number of the gene counts that were significantly enriched in the GO list, the dot color represents the p-adjusted value (padj $\leq 0.05$ ). 
b

d
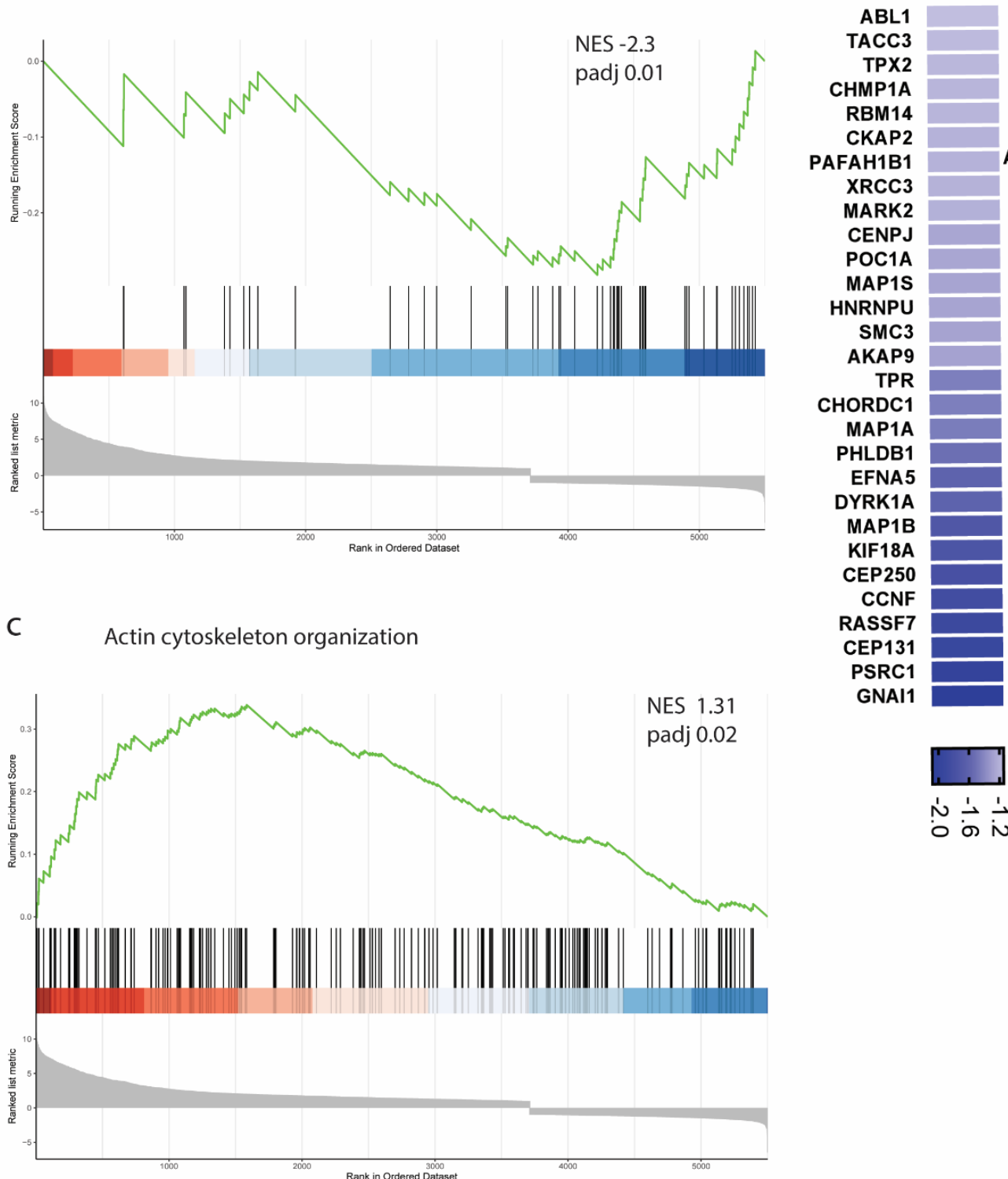

VIL1

ARHGEF15 AVIL

ACTA1

INA

MYBPC3

ARHGAP6

CAPN3

HCLS1

ADRA2A

EPHA1

SMTNL1

MYBPC2

HRG

SEMA3E

FSCN3

FRMD7

FGD5

SMTNL2

NEB

CDK5R1

CATIP

GMFG

KANK3

LMOD1

ESPNL

MYOM1

MYOZ1

TMOD4

in

TNXB

व்ं BST1

FGF7

BCL6

RND1

FES

PAK3

SERPINF2

TNF

GDPD2

GPR65

SPTBN5

LMOD2

SYNPO2L

MYOM3

ELMO1

PSTPIP2

LDB3

STAP1

LMOD3

PLEK

SPECC1

AIF1

ANKRD1

NPHS1

EPHA3

RHOH

POF1B

TRPV4

MYLK3

$\triangle \infty \infty$

\section{Figure 5}

GSEA plots for enriched genes regulating the cytoskeleton structure. a GSEA plot indicating the negative running enrichment score for the "regulation of microtubule cytoskeleton organization" (NES= -2.3 , padj= 0.01 ) $b$ with a number of the down-regulated genes participating in the process displayed in the form of a heatmap. c GSEA plot showing the positive running enrichment score activating the "actin cytoskeleton organization" (NES=1.31, padj= 0.02) d with a list of the top activated genes shown in a heatmap. Black 
bars underneath the graph present the rank positions of genes from the gene set. The green line refers to the enrichment profile.
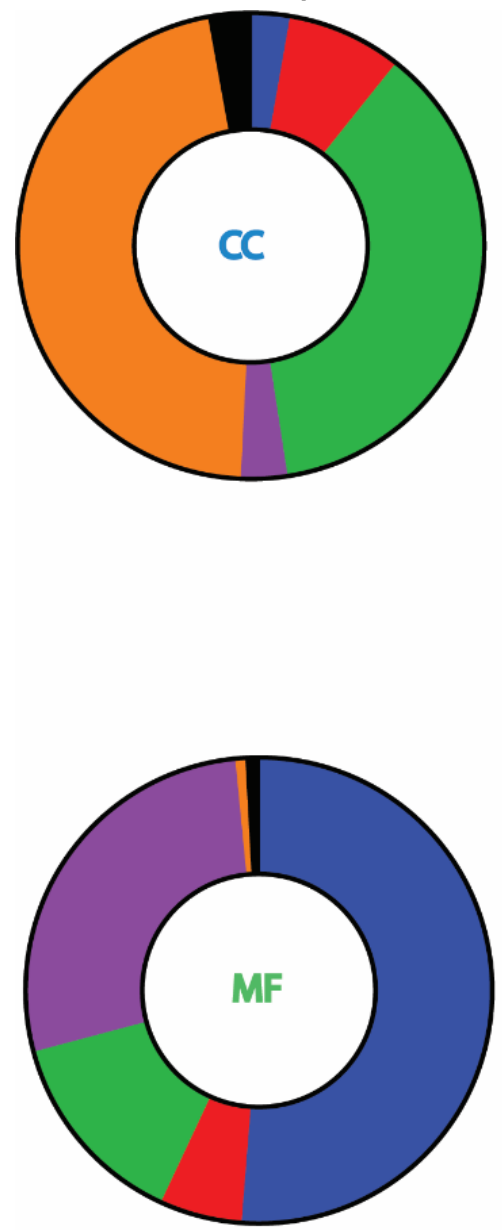

Ribosome

Integral compnent of plasma membrane

Chromosome, centromeric region

Intracellular

Cytosolic large ribosomal subunit

Cytosolic small ribosomal subunit

Viral transcription

Translational elongation

Translational termination

Negative regulation of viral genome replication

Viral life cycle

Nuclear-transcribed mRNA catabolic process, nonsense mediated decay

Metal ion binding

- Structural consistetuent of ribosome

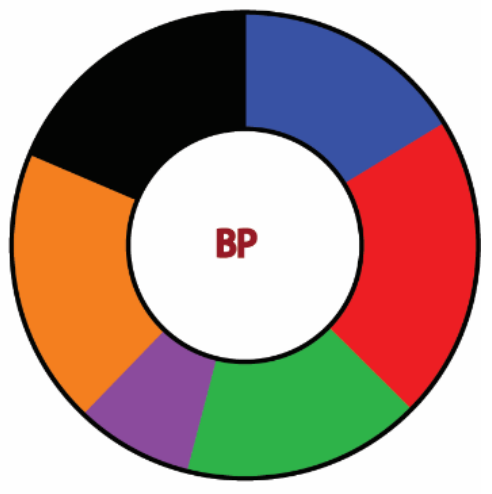

RNA binding

Nucleic acid biding

Transforming growth factor beta receptor binding

Hydrolase activity, acting on acid anhydrides

Figure 6

ORA gene ontology results from innateDB. Each pie chart represents the top $6 \mathrm{GO}$ terms of the (CC) cellular components, (BP) biological processes, and (MF) molecular functions (padj $\leq 0.05$ ). 


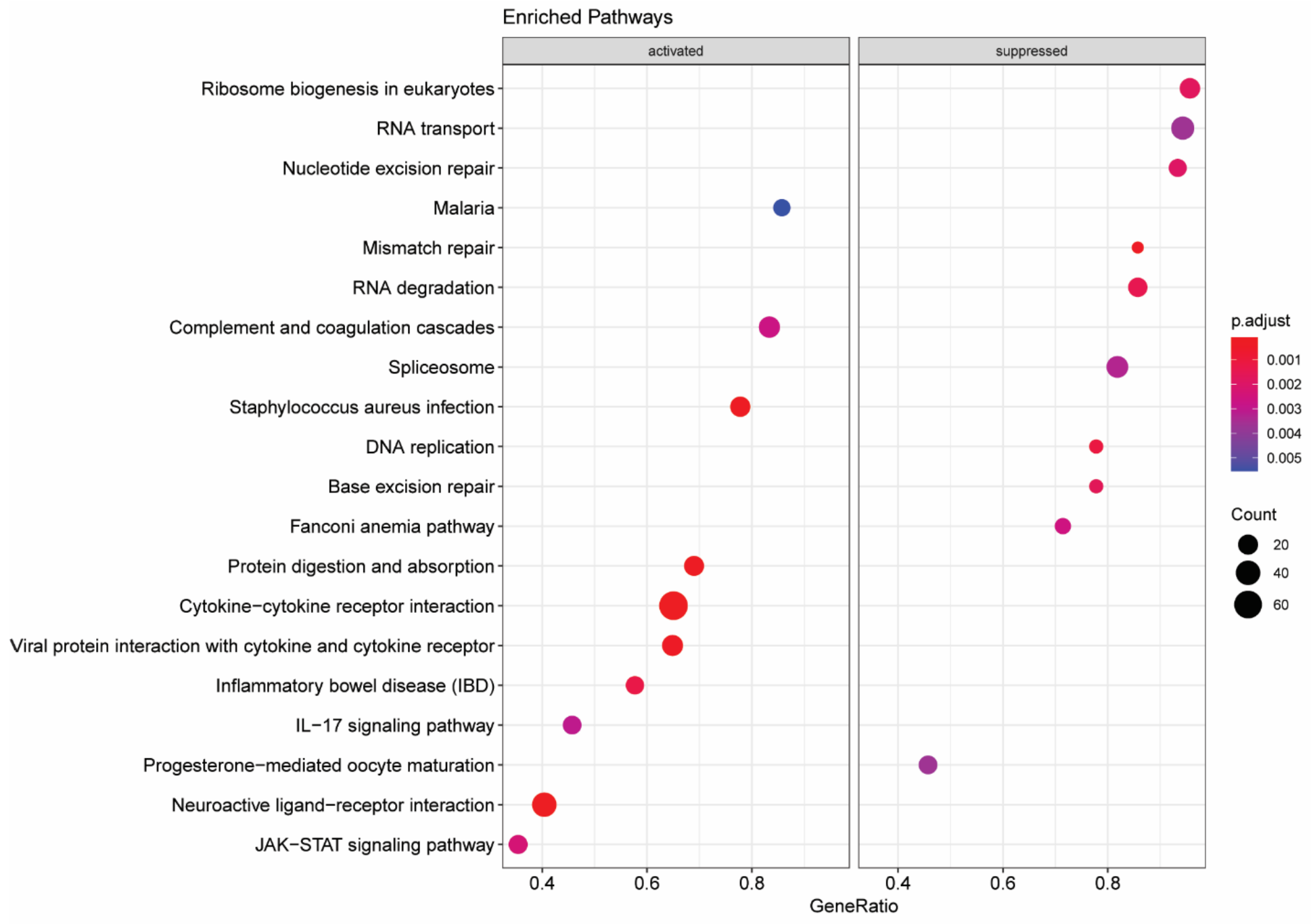

Figure 7

KEGG Pathway analysis. KEGG-based GSEA pathways are plotted in the order of gene ratios. The dots' size depicts the count number of the genes significantly differentiated in the KEGG pathway list, and the color represents the p-adjusted value (padj $\leq 0.05$ ). 


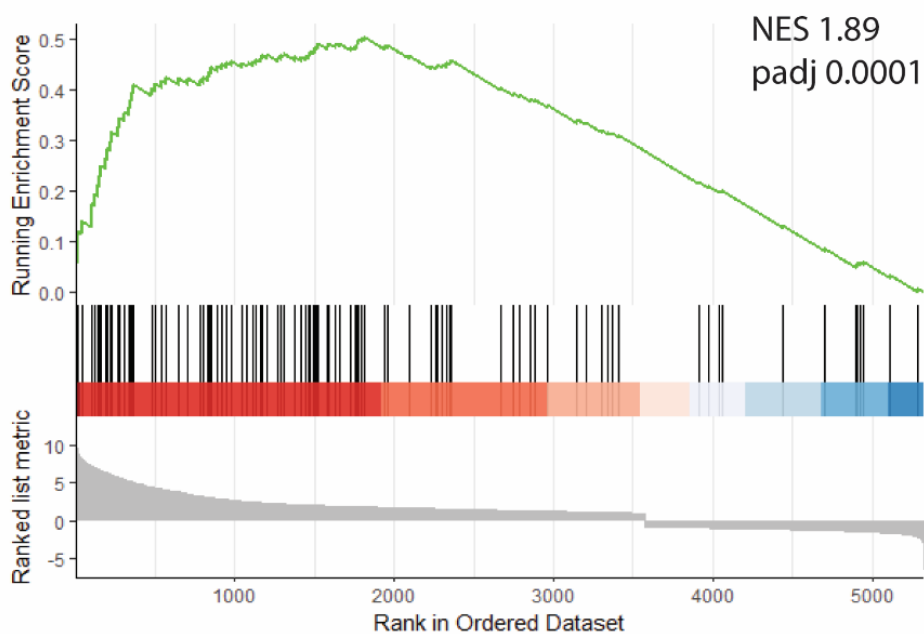

C Neuroactive ligand-receptor interaction

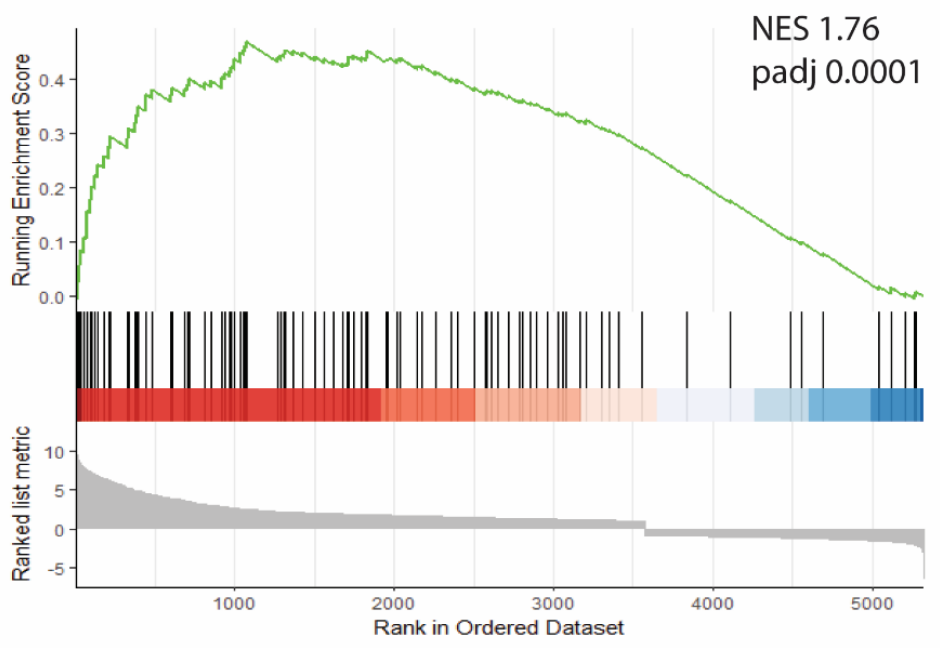

b

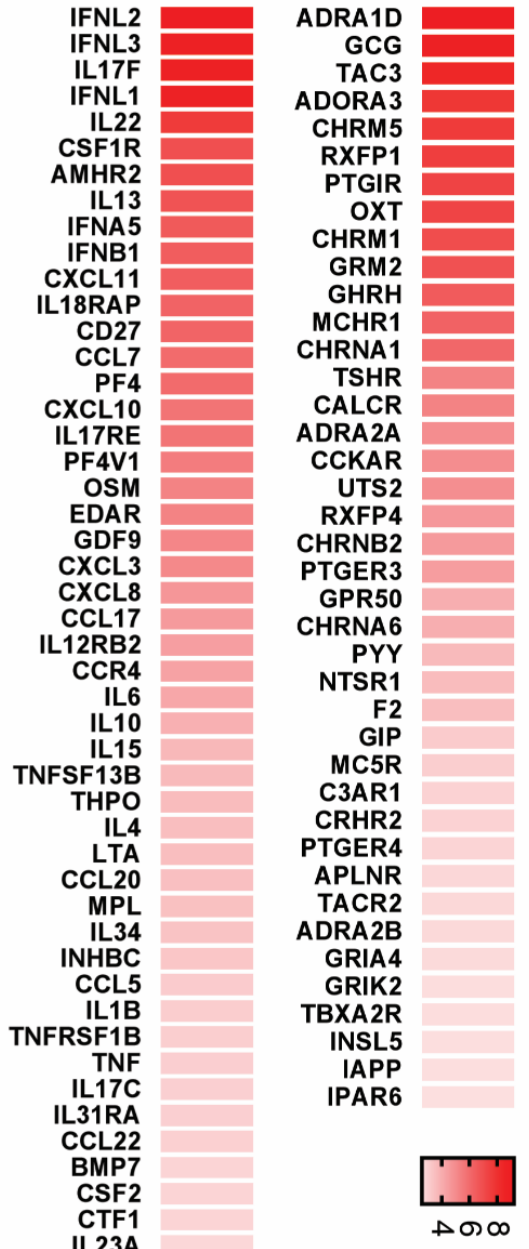

d

ADRA1D

HRM

GRM

MCHR1

MRNA

ADRA2A

CCKAR

RXNB

PTGER3

GIP

PTGER

NSL

IAPP

$\triangle \infty \infty$

CXCL2

TNFSF14

TNFRSF10D

CSF2RA

ACKR4

BMP8A

INHBE

NODAL

TNFRSF4

BMP8B

CD40LG

IL23R

ACVRL1

TNFRSF11

TNFRSF9

IFNLR1

TNFSF10

GDF5

CCR6

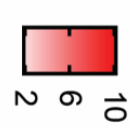

Figure 8

GSEA plots of enriched KEGG pathways. a GSEA plots indicating the running enrichment score for gene expression signature of 'cytokine-cytokine receptor interaction' (NES=1.89, padj=0.0001), and its associated top up-regaulted genes $b$ visualized in a heatmap, in addition to the GSEA plot for the $c$ 'neuroactive ligand-receptor interaction' pathway (NES=1.76, padj= 0.0001$)$ and its associated top activated genes $d$. Black bars underneath the graph present the rank positions of genes from the gene set, 
the green line refers to the enrichment profile. GO, KEGG and GSEA were performed by the R package clusterProfiler; R package DOSE; R package org.HS.eg.db. and visualized by the R package Enrichplot and $\mathrm{R}$ package ggplot2.

a

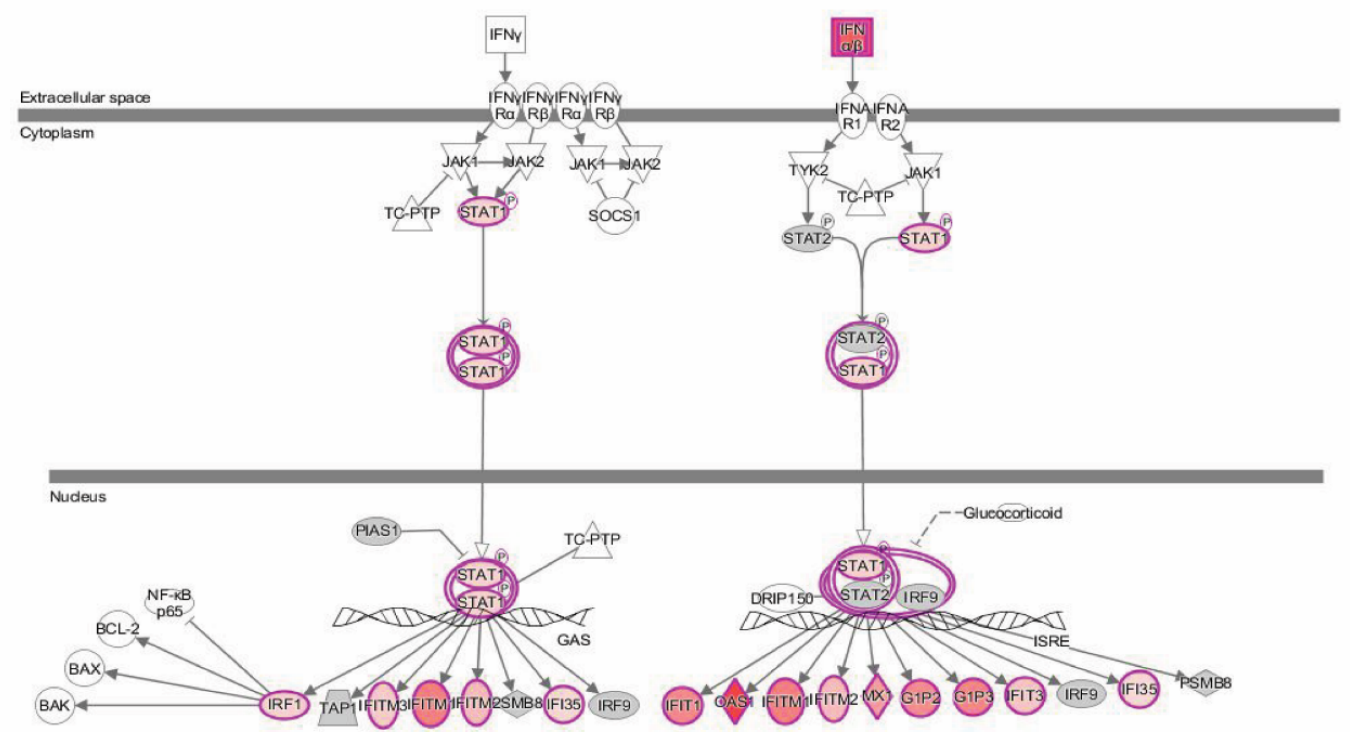

b

IFN-lambda $2 / 3$ C ISG15 e
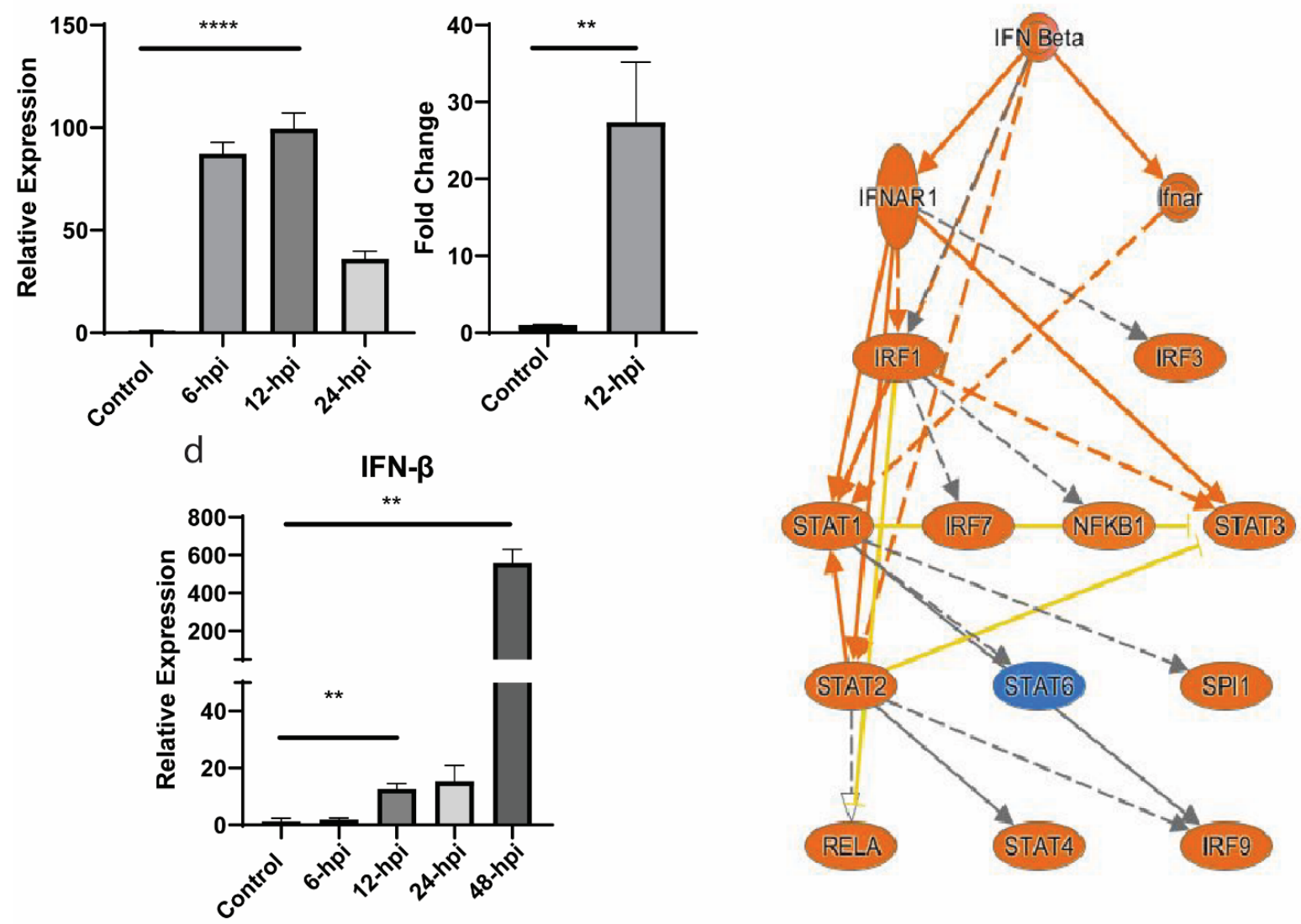

Figure 9

Regulation of induced immune genes expression in hBMECs following influenza A virus infection. a Canonical pathway of the "Interferon signaling" for the genes differentially expressed in hBMECs 12 hpi, 
genes that are significantly up-regulated are shown in red. The intensity of red corresponds to an increase in fold change levels of the cells infected with A/WSN/33 (H1N1) compared to the control cells zscore $=4.2$. White nodes specify genes with no significant gene expression at $12 \mathrm{hpi}$. The pathway was generated with IPA (Ingenuity pathways system). b quantification of gene expression by (RT-qPCR) for IFN- $\lambda$ 2/3 c ISG15, and d IFN- $\beta$. (b-d) the hBMECs were infected with A/WSN/33 (H1N1) at MOI 0.1, and the target genes were quantified by relative quantification $\mathrm{qPCR}$. statistical-significance analyzed by $\mathrm{t}$-test ${ }^{*} p<0.05, * \star p<0.01$, and ${ }^{\star \star \star \star} p<0.0001$. e Mechanistic network by IPA for the upstream regulators interacting with IFN- $\beta$, which enables to discover plausible sets of connected upstream regulators that can work together to elicit the gene expression changes observed in our dataset. 

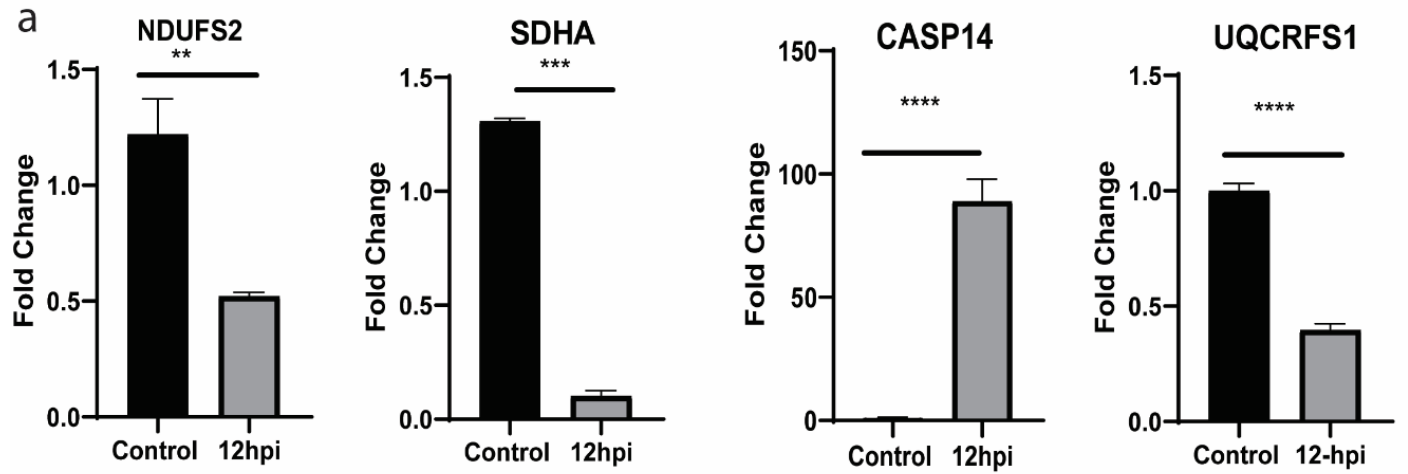

b Mitochondrial protein complex

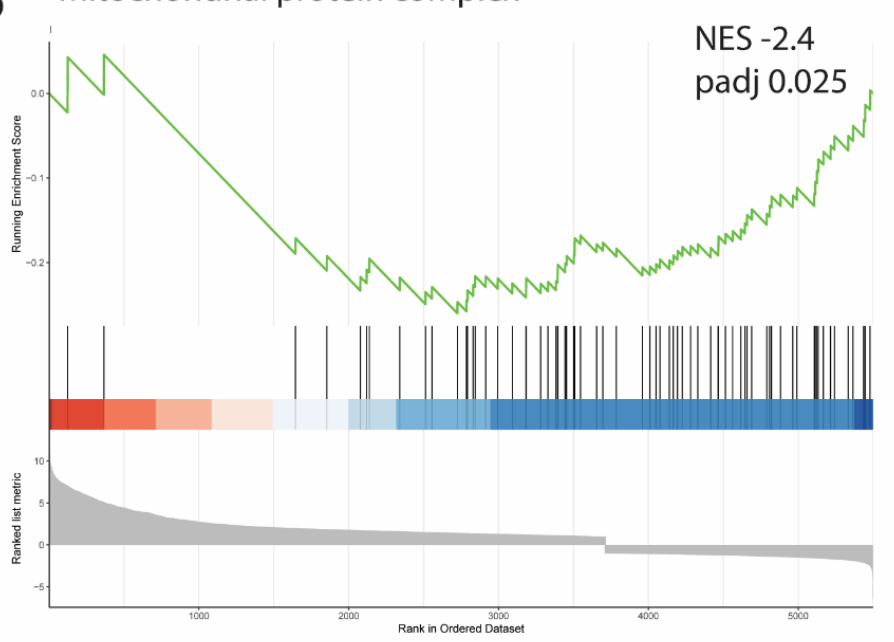

C

d Mitochondrial gene expression

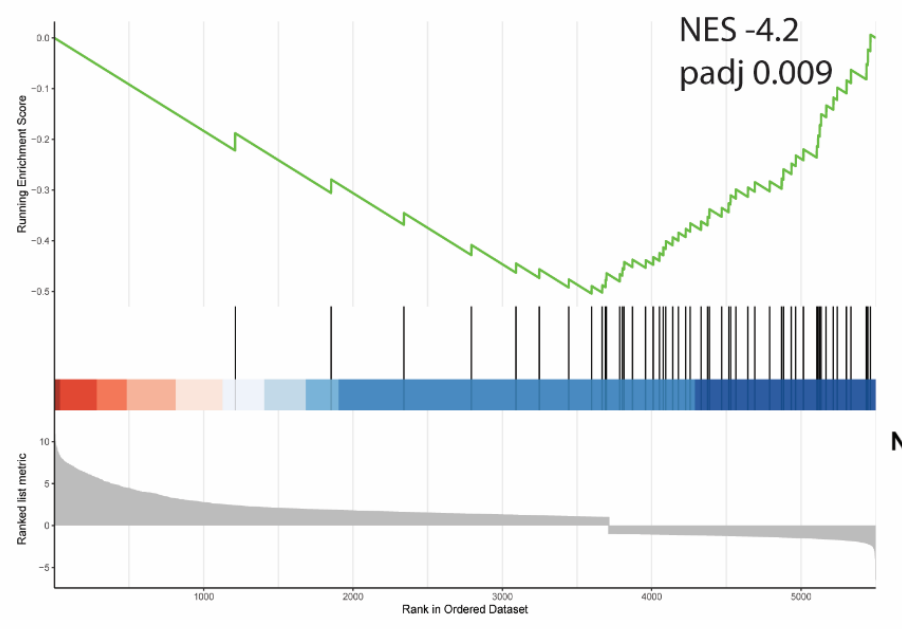

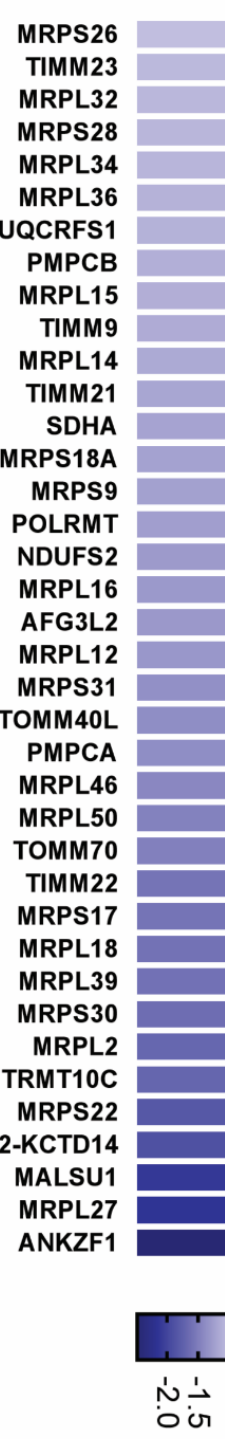

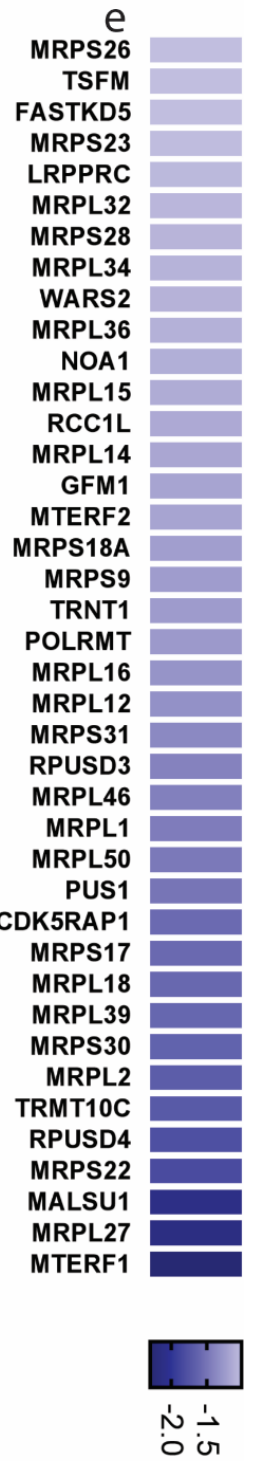

Figure 10

Altered mitochondrial gene expression of hBMECs. a quantification of gene expression by (RT-qPCR) showing the fold change increase or decrease for four mitochondrial genes expression NDUSF2, SDHA, CASP14, UQCRFS1 within the infected hBMECs cells at $12 \mathrm{hpi} \mathrm{compared} \mathrm{to} \mathrm{the} \mathrm{control} \mathrm{cells.} \mathrm{statistical-}$ significance analyzed by t-test * $p<0.05$, $* \star p<0.01$, $* \star \star p<0.001$, and $* \star \star \star p<0.0001$. b the GSEA running enrichment score for the suppression of 'mitochondrial protein complex' $(\mathrm{NES}=-2.4, \mathrm{padj}=0.025)$ 
enriched GO term and $\mathrm{c}$ its top downregulated genes. $d$ indicates the GESA plot for negative running enrichment score of 'mitochondrial gene expression' (NES=-4.2, padj=0.009) and e the top participating downregulated genes visualized in a heatmap. Black bars underneath the graph present the rank positions of genes from the gene set, the green line refers to the enrichment profile.

a

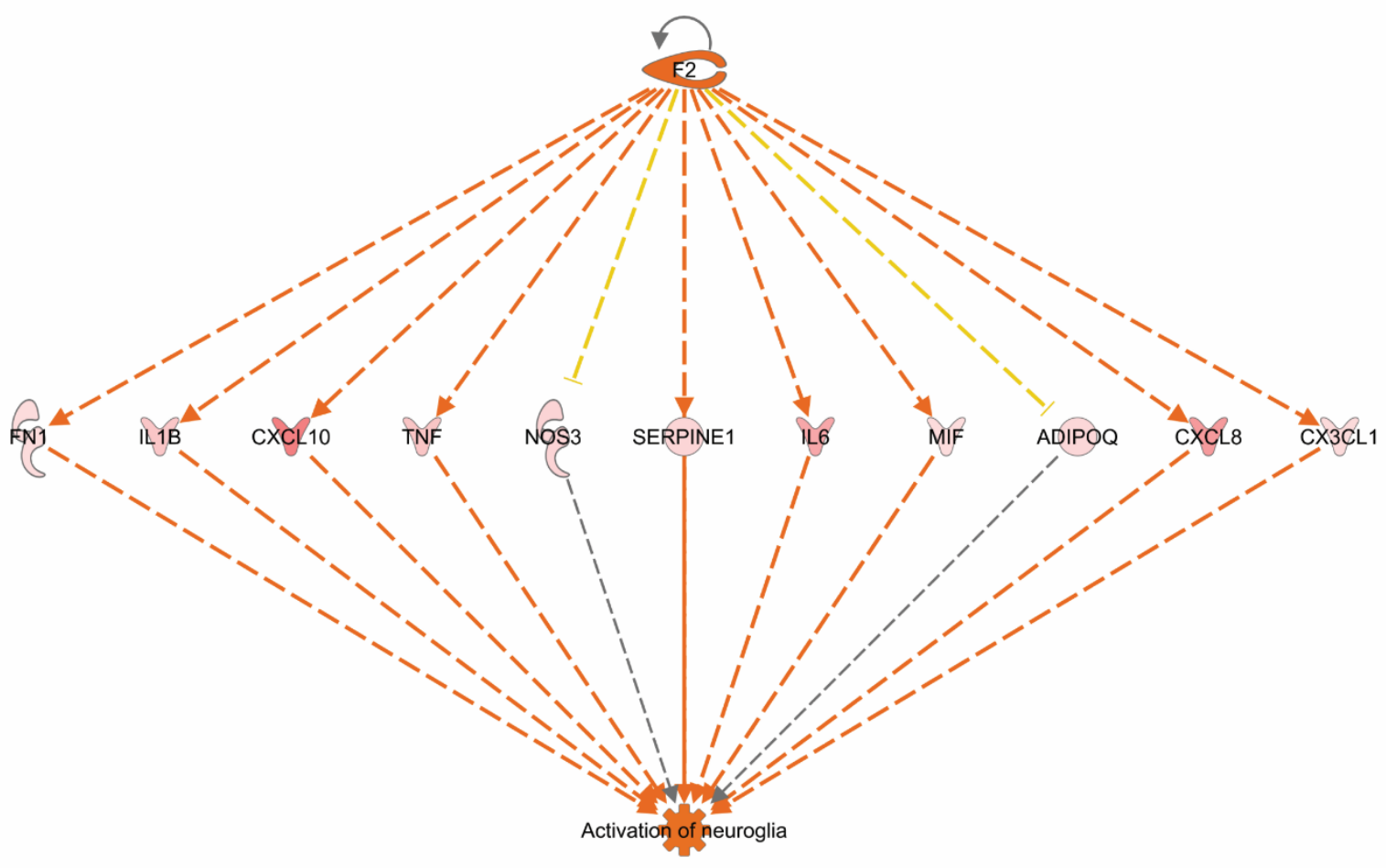

b

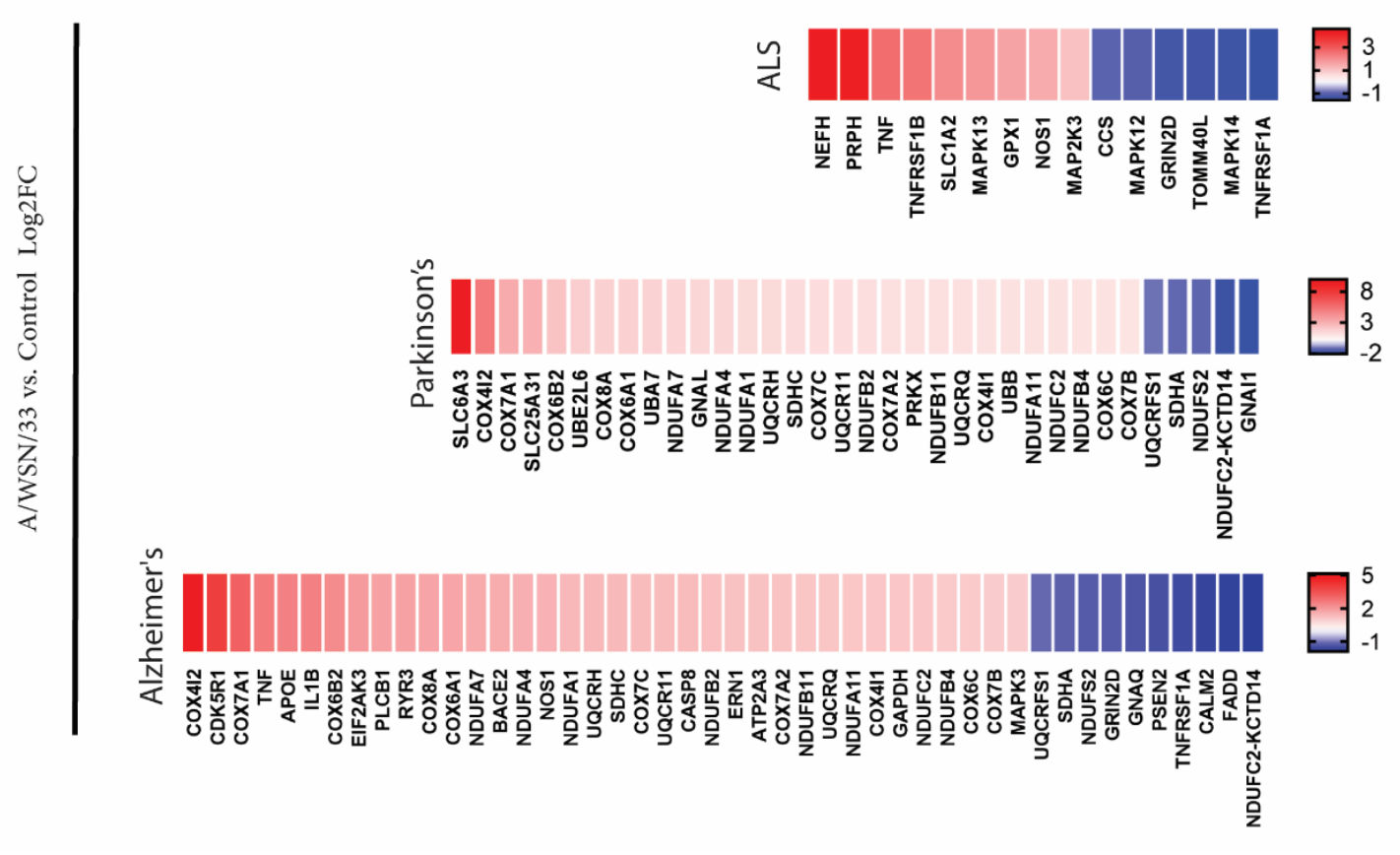

Figure 11 
Enrichment analysis indicating neurological disorders. a The IPA regulator effects analysis shows that the F2 upstream regulator and its downstream effects indicate a potential activation for the neuroglia in hBMECs at $12 \mathrm{hpi}$. The regulator effects algorithm generates hypotheses that explain how the activation or inhibition of regulators leads to an increase or decrease of function. $b$ the heatmaps reveal the genes participating in neurodegenerative disease pathways enriched and sorted according to Log2FC ratios in the DEGs list.

\section{Supplementary Files}

This is a list of supplementary files associated with this preprint. Click to download.

- Supplementaryfile1.pdf

- Supplementaryfile2.csv

- Supplementaryfile3.csv

- Supplementaryfile4.csv 